\title{
Inference of ecological and social drivers of human brain-size evolution
}

Mauricio González-Forero ${ }^{1 *}$ \& Andy Gardner ${ }^{1}$

${ }^{1}$ School of Biology, University of St Andrews, Dyers Brae, St Andrews KY16 9TH, UK

${ }^{*}$ Corresponding author

The human brain is unusually large. It tripled in size from Australopithecines to modern humans ${ }^{1}$ and became almost six times larger than expected for a placental mammal of human size ${ }^{2}$. Brains incur high metabolic costs ${ }^{3}$ and accordingly a long-standing question is why the large human brain evolved ${ }^{4}$. The leading hypotheses propose benefits of improved cognition for overcoming ecological ${ }^{5-7}$, social ${ }^{8-10}$, or cultural ${ }^{11-14}$ chal- $^{-}$ lenges. However, these hypotheses are typically assessed using correlative analyses, and establishing causes for brain-size evolution remains difficult ${ }^{15,16}$. Here we introduce a metabolic approach that enables causal assessment of social hypotheses for brain-size evolution. Our approach yields quantitative predictions for brain and body size from formalised social hypotheses given empirical estimates of the brain's metabolic costs. Our model predicts the evolution of adult Homo sapiens sized brains and bodies when individuals face a combination of $60 \%$ ecological, $30 \%$ cooperative, and $10 \%$ between-group competitive challenges, and suggests that between-individual competition has been unimportant. Moreover, our model indicates that brain expansion in Homo was driven by ecological rather than social challenges, perhaps strongly promoted by culture. Our metabolic approach thus allows for causal assessments that refine, refute, and unify hypotheses for brain-size evolution. 
The leading hypotheses for the evolution of brain size make different suggestions as to which cognitive challenges have been most important in driving brain expansion. "Ecologicalintelligence" hypotheses emphasize challenges posed by the non-social environment: e.g., food finding, caching, or processing ${ }^{5-7}$ (Fig. 1). In contrast, "social-intelligence" hypotheses emphasize challenges posed by the social environment: e.g., cooperating for resource extraction $^{10,15}$, manipulating others, avoiding manipulation or forming coalitions and alliances to out-compete others ${ }^{8,9}$ (Fig. 1). Social challenges have been suggested to constitute particularly powerful drivers of brain expansion because they may have triggered evolutionary arms races in cognition ${ }^{8-10}$. Finally, "cultural-intelligence" hypotheses emphasize challenges of learning from others, teaching, and doing so with accumulated cultural knowledge $\mathrm{e}^{11-14}$. Empirical tests of these hypotheses customarily investigate phylogeneticallycontrolled correlations between brain size (or the size of brain components) and candidate selective factors (e.g., diet type $\mathrm{e}^{5,17}$, tactical-deception rate ${ }^{18}$, group size $\mathrm{e}^{10,19}$, and sociallearning proclivity $\left.{ }^{20}\right)$. However, establishing causality has proven difficult: for example, given a positive correlation, it is unclear whether large group sizes favour bigger brains or big brains enable larger group sizes ${ }^{16}$. Moreover, there is the quantitative problem of explaining not only why bigger brains are favoured, but also why they are favoured to the particularly large size observed in humans $\left(\approx 1.3 \mathrm{~kg}\right.$ for a body size of $\approx 50 \mathrm{~kg}$ in females $\left.{ }^{21,22}\right)$.

To address these problems, we merge elements of metabolic theory ${ }^{23}$, life-history theory, and differential games to obtain quantitative predictions for the evolution of brain and body size when individuals face ecological and social challenges given brain metabolic costs. Our approach incorporates social interactions into a previous non-social model $^{24}$ (Supplementary Information sections 1-3). We consider a female population and partition the body mass of each individual into three tissues: "brain", "reproductive", and other "somatic" tis- 
sue. Part of the reproductive tissue's energy consumption is for production and maintenance of offspring, while part of brain's energy consumption is for production (learning) and maintenance (memory) of energy-extraction skills. Accordingly, at each age the individual has a certain skill level measured in information units (i.e., bytes). She extracts energy by using her skill level to overcome ecological or social energy-extraction challenges. Success in an ecological challenge depends on her own skill level, while success in a social challenge depends on her skill level and that of her social partners. We consider three types of social challenge: cooperative, in which the individual's skill level and that of a social partner of the same age (hereafter "peer") interact to overcome a challenge; between-individual competitive, in which the individual uses her skill level against that of a peer to extract energy; and between-group competitive, in which the individual's skill level and that of a peer act together and against the skills of another two peers (i.e., one coalition competing against another). We assume that during any small time interval, the individual faces energy-extraction challenges, a proportion $P_{j}$ of which are of type $j$ (collectively denoted by $\mathbf{P}$, with $j=1, \ldots, 4$ respectively indexing the four challenge types and $\left.\sum_{j=1}^{4} P_{j}=1\right)$. Given $\mathbf{P}$, the growth strategy controls the amount of energy allocated to the production of each tissue throughout life, and we let the growth strategy evolve. The individual's energy-extraction efficiency (EEE) thus depends on her skill level, that of social partners, and the challenges faced, and we consider two shapes for EEE (Fig. 2 and Extended Data Fig. 1). We use previously-published data for parameter estimates, including brain metabolic costs (Methods; Extended Data Figs. 2 and $3)$.

We find that increasing the proportion of cooperative challenges decreases both adult absolute brain size (hereafter "brain size") and adult relative brain size (hereafter "encephalization quotient" or "EQ": adult brain size divided by expected brain size for a given body 
size; Fig. 3a,b and Extended Data Fig. 4). In contrast, increasing the proportion of betweenindividual competitive challenges increases brain size if EEE is weakly-decelerating with skill (Fig. 3a), but decreases brain size if EEE is strongly-decelerating (Fig. 3b and Extended Data Fig. 4). However, although between-individual competition increases brain size with weakly-decelerating EEE, the result is larger brains and smaller bodies than those of modern humans (Fig. 3a and Extended Data Fig. 4). Between-individual competition also decreases body mass as it increases the difficulty of energy extraction and thus limits the energy available for body growth; consequently, between-individual competition increases EQ because body size decreases more strongly than brain size when the latter decreases. Increasing the proportion of between-group competition decreases brain size but increases EQ because body size decreases more strongly than brain size [with weakly-decelerating EEE and submultiplicative cooperation (Figs. 3a); in the other cases, between-group competition has similar effects as between-individual competition (Extended Data Fig. 4)]. Moderatelyfrequent between-individual or between-group competition can lead to no allocation to brain and body growth (blue dots in Fig. 3a and Extended Data Fig. 4, and Extended Data Fig. 5a,d); additionally, moderately-frequent between-group competition in the presence of substantial cooperation can lead to arms races in brain size, which fail to yield stable, large brains [e.g., due to cycling in brain size or eventual collapse to no allocation into brain growth (Extended Data Fig. 5)]. This is because energy extraction becomes exceedingly difficult in the presence of large-brained competitors such that investments in brain or body growth do not pay off.

To determine if any combination of social-challenge parameters $\mathbf{P}$ yields an accurate prediction of adult brain and body sizes of $H$. sapiens and closely-related species, we obtained solutions exhaustively across the $\mathbf{P}$-parameter space while holding the other param- 
eters ( $\mathbf{Q}$ and $\mathbf{R}$ ) fixed (Fig. 3d,e; Supplementary Information section 5). We find near-perfect adult fits across Homo species (Fig. 4a and Extended Data Figs. 6-8). A near-perfect adult fit for $H$. sapiens occurs with a large proportion of ecological challenges ( $\approx 60 \%$ ), a moderate proportion of cooperative challenges $(\approx 30 \%)$, a small proportion of between-group competitive challenges $(\approx 10 \%)$, and a virtual absence of between-individual competitive challenges $(\approx 0 \%)$ (Figs. 3e and $4 \mathrm{a}$ and Extended Data Figs. 6 and 9 ). In the resulting reconstruction, the proportion of ecological challenges tends to increase from early to late Homo, and a steep increase in EQ from H. ergaster to H. heidelbergensis is due to a transition from strongly- to weakly-decelerating EEE (Fig. 4a). The adult best-fit eco-social scenario for $H$. sapiens also yields a predicted life history that closely matches the species' life-history timing (Fig. 4b and Extended Data Fig. 10). The resulting ontogenetic fit is high for body size, but lower for brain size early in ontogeny (Fig. 4b), perhaps due in part to our use of a power-law relationship between resting metabolic rate and body mass that underestimates resting metabolic rate early in ontogeny ${ }^{24}$. With the adult brain size resulting from the bestfitting scenario for $H$. sapiens $\left[x_{\mathrm{b}}^{*}\left(t_{\mathrm{a}}\right)=1.276 \mathrm{~kg}\right.$, the predicted adult skill level for energy extraction is $3.92 \mathrm{~TB}$, which can be calculated with an equation transforming brain mass to skill level $^{24}\left[\hat{x}_{\mathrm{k}}=s_{\mathrm{k}} B_{\mathrm{b}} x_{\mathrm{b}}^{*}\left(t_{\mathrm{a}}\right) / B_{\mathrm{k}}\right.$ where $\hat{x}_{\mathrm{k}}$ is the asymptotic skill level in adulthood; Eq. (5) in Methods]. For comparison, current rough estimates suggest a human-neocortex storage capacity of $\approx 600 \mathrm{~TB}$ (Supplementary Information section 4.3$)^{25}$.

Using previously-published data for parameter estimates, our results suggest that adult human-sized brains and bodies may result from ecological challenges as drivers of brain expansion, with cooperation and between-group competition decreasing brain and body size and between-group competition increasing EQ by decreasing body size more strongly than brain size (Fig. 3a and Extended Data Fig. 4b). In this eco-social scenario, between- 
individual competition plays little role as it does not lead to human-sized brains and bodies. Cooperation decreases brain size because it allows individuals to rely on their partners' skills and thus decrease their own investment into costly brains (cooperation invites cheating), which is consistent with diminished brain sizes in cooperatively-breeding birds ${ }^{26}$ and mammals ${ }^{27}$, including primates ${ }^{28}$. For instance, among mole rats, naked mole rats are the most specialized in cooperative breeding and have the smallest relative brain size ${ }^{29}$ (however, allomaternal care and brain size are positively associated in mammals ${ }^{30}$, but allomaternal care constitutes cooperation targeted at young vanishing in adulthood as opposed to the peer-cooperation studied here). Similarly, between-group competition can decrease brain size probably because between-group competition involves cooperation between group members, allowing individuals to rely on their partners' skill. The result that exceedingly-frequent competition decreases absolute and relative brain size may be relevant to the observed decreased brain size in cetaceans with the largest group sizes ${ }^{19}$. Cooperation can also decrease body size in our model because when brain size is disfavoured so too can be body size. This is because a consequence of our model is that a key reason to grow somatic tissue is to make energy available for brain growth: increasing the mass of inexpensive somatic tissue can increase the energy available for tissue (and brain) growth due to the physical constraint imposed by the power-law relationship between resting metabolic rate and body mass $^{24}$.

Overall, our assessment fails to support social hypotheses as explanations for the evolution of human brain size, and is more consistent with ecological hypotheses. Our results suggest causal interpretations that differ from some current thinking on the evolution of human cognition. Specifically, we obtained an eco-social scenario that involves a substantial proportion of cooperation (30\% against nature and $10 \%$ against others), which could shape 
cognition towards cooperation. This would help explain aspects of human cognition that facilitate cooperation $^{11}$, even if cooperation has not been a driver of human brain expansion. Additionally, since our analysis suggests that brain expansion in Homo has not been driven by peer cooperation or competition, our results may suggest that social complexity is a consequence rather than a cause for the human brain size — although we emphasize that our analysis is an illustrative starting point and future extensions are encouraged (see Supplementary Information section 9). Therefore, our results highlight the fundamental question of why ecological challenges would have favoured substantial brain expansion in humans but less so in other taxa ${ }^{10,15}$. One clue is suggested by our finding that $H$. sapiens-sized brains and bodies obtain only under weakly-decelerating EEE (Figs. 3, 4 and Extended Data Fig. 6a): in other words, only when young individuals can maintain a substantial rate of increase in their efficiency of energy extraction as they acquire skills. One possibility is that culture (or cumulative culture) facilitates weakly-decelerating EEE if learning from the population's pool of skills allows individuals to maintain a relatively high rate of increase in EEE as their skill level increases when young. More specifically, the evolution of progressively-elaborated social learning, teaching, and language ${ }^{11-14}$ may have enabled young individuals to continue gaining skills with age, possibly promoting less strongly decelerating EEE. In this respect, our results are consistent with aspects of various cultural hypotheses for brain evolution ${ }^{13,14}$ and an explicit account of the effect of culture on EEE could help address whether culture (or cumulative culture) has enabled ecological challenges to drive brain expansion in humans in ways that have not occurred in other taxa.

1. Klein, R. G. The Human Career (The Univ. of Chicago Press, 2009), 3rd edn.

2. Martin, R. D. Relative brain size and basal metabolic rate in terrestrial vertebrates. $\mathrm{Na}$ - 
ture 293, 57-60 (1981).

3. Aiello, L. C. \& Wheeler, P. The expensive-tissue hypothesis: the brain and the digestive system in human and primate evolution. Curr. Anthropol. 36, 199-221 (1995).

4. Darwin, C. The Descent of Man, and Selection in Relation to Sex (J. Murray, 1871).

5. Clutton-Brock, T. H. \& Harvey, P. H. Primates, brains and ecology. J. Zool. 190, 309-323 (1980).

6. Gibson, K. R. Cognition, brain size and the extraction of embedded food resources. In Else, J. G. \& Lee, P. C. (eds.) Primate Ontogeny, Cognition and Social Behaviour, 93-103 (Cambridge Univ. Press, 1986).

7. Rosati, A. G. Foraging cognition: reviving the ecological intelligence hypothesis. Trends Cogn Sci 21, 691-702 (2017).

8. Humphrey, N. K. The social function of the intellect. In Bateson, P. P. G. \& Hinde, R. A. (eds.) Growing Points in Ethology, 303-317 (Cambridge Univ. Press, 1976).

9. Byrne, R. \& Whiten, A. (eds.) Machiavellian Intelligence (Oxford Univ. Press, 1988).

10. Dunbar, R. I. M. The social brain hypothesis. Evol. Anthropol. 6, 178-190 (1998).

11. Moll, H. \& Tomasello, M. Cooperation and human cognition: the Vygotskian intelligence hypothesis. Phil. Trans. R. Soc. B 362, 639-648 (2007).

12. van Schaik, C. P. \& Burkart, J. M. Social learning and evolution: the cultural intelligence hypothesis. Phil. Trans. R. Soc. B 366, 1008-1016 (2011).

13. Henrich, J. The Secret of our Success (Princeton Univ. Press, 2016).

14. Laland, K. N. Darwin's Unfinished Symphony (Princeton Univ. Press, 2017). 
15. Dunbar, R. I. M. \& Shultz, S. Why are there so many explanations for primate brain evolution? Phil. Trans. R. Soc. B 372, 20160244 (2017).

16. Powell, L. E., Isler, K. \& Barton, R. A. Re-evaluating the link between brain size and behavioural ecology in primates. Proc. R. Soc. B 284, 20171765 (2017).

17. DeCasien, A. R., Williams, S. A. \& Higham, J. P. Primate brain size is predicted by diet but not sociality. Nat. Ecol. Evol. 1, 0112 (2017).

18. Byrne, R. W. \& Corp, N. Neocortex size predicts deception rate in primates. Proc. R. Soc. B 271, 1693-1699 (2004).

19. Fox, K. C. R., Muthukrishna, M. \& Shultz, S. The social and cultural roots of whale and dolphin brains. Nat. Ecol. Evol. 1, 1699-1705 (2017).

20. Street, S. E., Navarrete, A. F., Reader, S. M. \& Laland, K. N. Coevolution of cultural intelligence, extended life history, sociality, and brain size in primates. Proc. Natl. Acad. Sci. USA 114, 7908-7914 (2017).

21. Kuzawa, C. W. et al. Metabolic costs and evolutionary implications of human brain development. Proc. Nat. Acad. Sci. USA 111, 13010-13015 (2014).

22. Dekaban, A. S. \& Sadowsky, D. Changes in brain weights during the span of human life: relation of brain weights to body heights and body weights. Ann. Neurol. 4, 345-356 (1978).

23. West, G. B., Brown, J. H. \& Enquist, B. J. A general model for ontogenetic growth. Nature 413, 628-631 (2001).

24. González-Forero, M., Faulwasser, T. \& Lehmann, L. A model for brain life history evolution. PLoS Comput. Biol. 13, e1005380 (2017). 
25. Bartol Jr, T. M. et al. Nanoconnectomic upper bound on the variability of synaptic plasticity. eLife 4, e10778 (2015).

26. Fedorova, N., Evans, C. L. \& Byrne, R. W. Living in stable social groups is associated with reduced brain size in woodpeckers (Picidae). Biol. Lett. 13, 20170008 (2017).

27. Clutton-Brock, T. Mammal Societies (Wiley, 2016).

28. Thornton, A. \& McAuliffe, K. Cognitive consequences of cooperative breeding? A critical appraisal. J. Zool. 295, 12-22 (2015).

29. Kruska, D. C. T. \& Steffen, K. Encephalization of Bathyergidae and comparison of brain structure volumes between the zambian mole-rat Fukomys anselli and the giant molerat Fukomys mechowii. Mamm. Biol. 74, 298-307 (2009).

30. Isler, K. \& van Schaik, C. P. Allomaternal care, life history and brain size evolution in mammals. J. Hum. Evol. 63, 52-63 (2012).

Supplementary Information is available in the online version of the paper.

Acknowledgements We thank Laurent Lehmann for advice in the model set up, and for support at the University of Lausanne where this project initiated. We also thank Piret Avila, Stephen H. Montgomery, Simon B. Laughlin, Maurizio Falcone, Tim Clutton-Brock, and Michael B. Morrissey for discussions, and Andrew Whiten for feedback. MGF was funded by a Marie Skłodowska-Curie Individual Fellowship (No 701464) and AG was funded by a NERC Independent Research Fellowship (NE/ K009524/1). The contents of this paper reflect only the authors' view and not the views of their funders.

Author Contributions MGF conceived and carried out the study. AG conceived implementing challenge proportions during small time intervals and how to obtain high fit intervals. MGF and AG wrote 
the paper.

Author Information Reprints and permissions information is available at www.nature.com/reprints.

The authors declare no competing financial interests. Correspondence and requests for materials should be addressed to MGF (mgf3@st-andrews.ac.uk). 


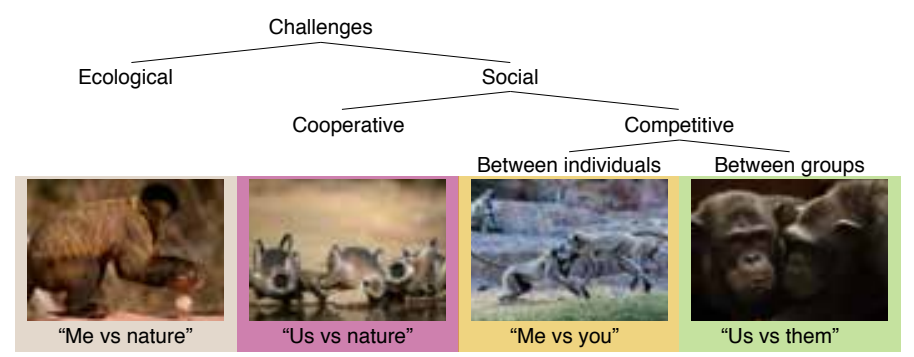

Figure 1: Ecological and social hypotheses for brain expansion. Ecological hypotheses emphasize challenges "against nature", while social hypotheses emphasize challenges involving social partners. Here we partition these hypotheses into four types of challenges that are expected to trigger different evolutionary processes.

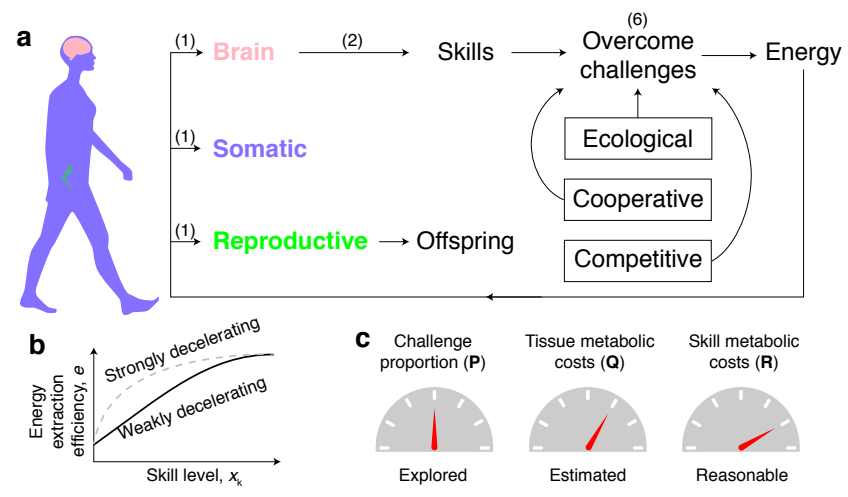

Figure 2: Model description. a, Schematic description of the model (see text for details). Parenthetical values refer to the corresponding equations (Methods). The model depends on $\mathbf{b}$, the shape of EEE with respect to skill and $\mathbf{c}$, three sets of parameters $\mathbf{P}, \mathbf{Q}$, and $\mathbf{R}$. 


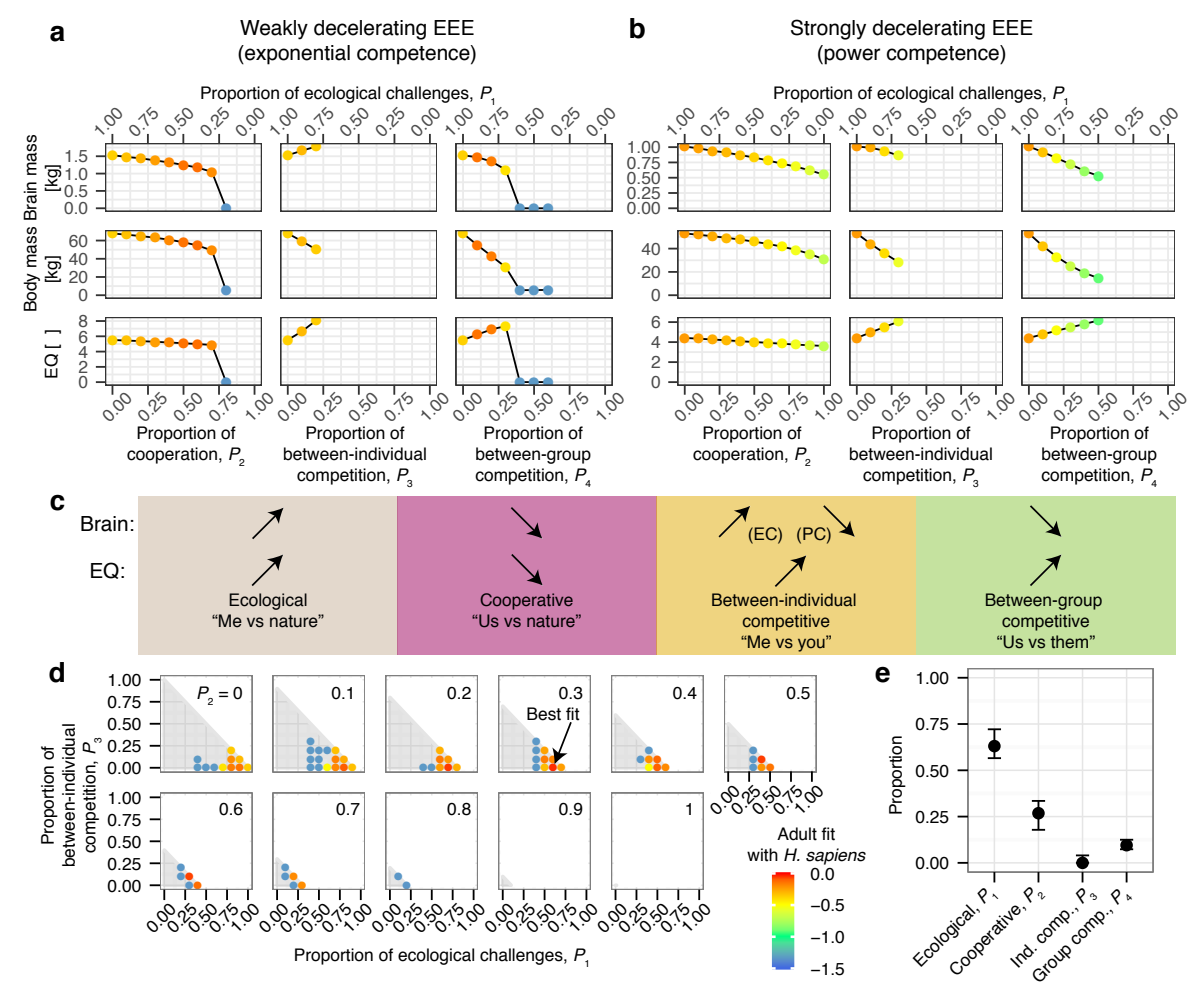

Figure 3: Effects of challenge types on brain size, and best-fitting scenario for adult

H. sapiens. a-b, Effects of increasing the proportion of a challenge type while decreasing the proportion of ecological challenges. For a, weakly-decelerating EEE (exponential competence) and $\mathbf{b}$, strongly-decelerating EEE (power competence). Dot colour is the adult fit with $H$. sapiens' brain and body mass [i.e., $-D\left(\tau_{\mathrm{a}}\right)$; Supplementary Information section 6 ]. Zero adult fit means perfect fit. c, Qualitative effects of challenge types on brain mass and EQ. d, Best fitting scenario for H. sapiens. Dots give the adult fit for every challenge combination that was solvable. Shaded regions indicate the simplex where $P_{j}$ can vary. The best fit occurs in $\mathbf{P}^{*}=(0.6,0.3,0,0.1)$ (adult fit: -0.03$)$. e, "High fit" intervals around $\mathbf{P}^{*}$ where adult fit is greater than -0.05 and dots are interpolated points with the best fit. 


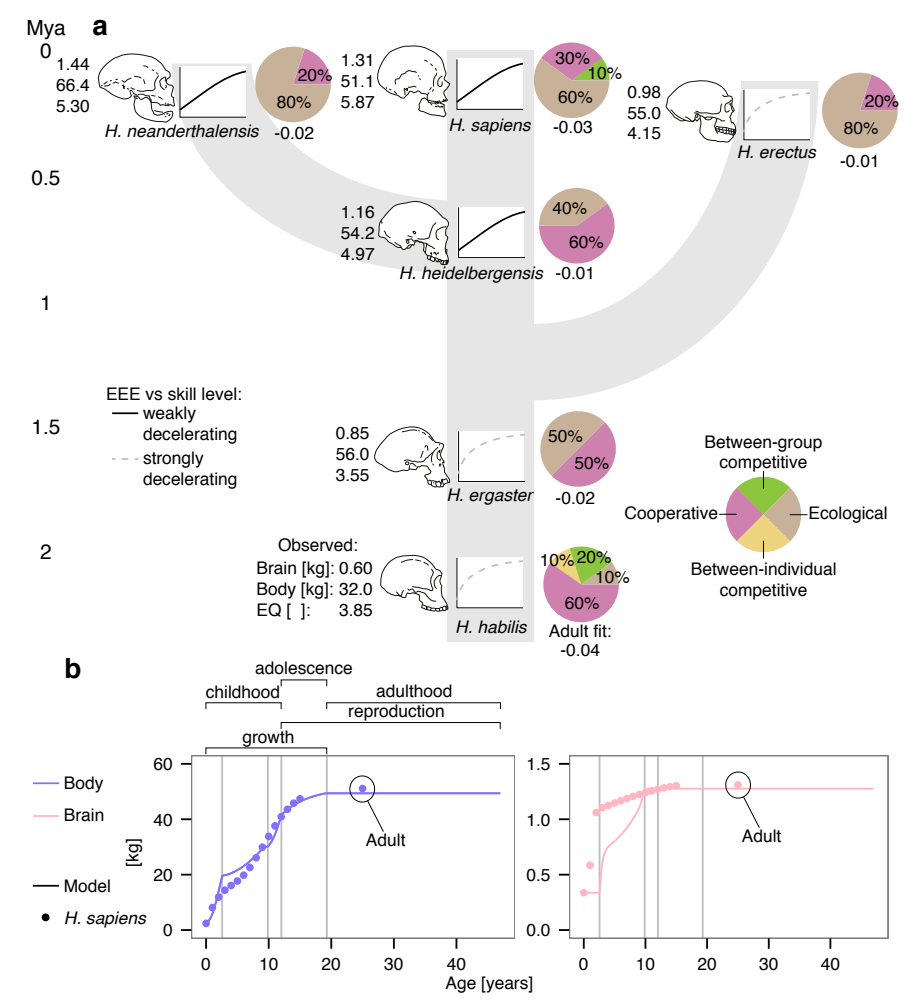

Figure 4: Best-fitting scenarios across adult Homo, and resulting predicted life-history for

H. sapiens. a, Best adult fitting scenarios across Homo (figure modified with permission from Fig. 8.1 of ref. $^{1}$ ). Pie charts and next-standing plots give respectively the challenge combination and shape of EEE vs skill that yield the best adult fit using the same $\mathbf{Q}$ and $\mathbf{R}$ parameters (Extended Data Figs. 6 and 7). b, Life history with the challenge combination yielding the best adult fit for $H$. sapiens. Resulting life periods are on top of the body mass plots. Vertical lines are ages where the growth strategy changes suddenly; within childhood, they occur when brain growth begins and terminates. 


\section{Methods}

Here we summarize our model; see Supplementary Information for the detailed method description. No experiments were performed for this study, and no empirical data were collected.

Model description. We consider a female population with overlapping generations and partition the body mass of each individual into three tissues: "brain", "reproductive", and other "somatic" tissue. Each individual extracts energy from the environment at each time to grow and maintain her tissues. We assume that some of the energy consumed by reproductive tissue is for production and maintenance of offspring, while some of that consumed by brain tissue is for production (learning) and maintenance (memory) of domain-general, energyextraction skills. Accordingly, at each age the individual has a certain skill level measured in information units (i.e., bytes). She extracts energy by using her skill level to overcome energy-extraction challenges that can be ecological or social. Success in an ecological challenge depends on her own skill level and on the challenge difficulty which is determined by the (non-evolving) environment. In contrast, success in a social challenge depends on her skill level and that of her social partners. We consider three different types of social challenge: cooperative challenges, in which the individual's skill level and that of a social partner of the same age ("peer") interact to overcome a challenge whose difficulty is determined by the environment; between-individual competitive challenges, in which the individual uses her skill level against that of a peer to extract energy, such that the difficulty of energy extraction is determined by her competitor's skill level; and between-group competitive challenges, in which the individual's skill level and that of a peer act together and against the skills of another two peers (i.e., one coalition competing against another), which deter- 
mines the challenge difficulty. During any small time interval, the individual faces challenges, a proportion $P_{j}$ of which are of type $j$ (with $j=1, \ldots, 4$ respectively indexing the four challenge types and $\sum_{j=1}^{4} P_{j}=1$ ). For instance, $P_{1}=1$ denotes that individuals face only ecological challenges, while $P_{1}=P_{2}=0.5$ denotes that individuals face only ecological and cooperative challenges and with equal proportion. We define the growth-metabolic rate as the rate of heat release by a resting individual due to tissue production. Moreover, we define the growth strategy as the fraction of the growth-metabolic rate due to the production of each tissue throughout life. Thus, the growth strategy generates an ontogenetic profile of brain and body size. We consider that the growth strategy evolves by natural selection, and study its evolution using standard evolutionary-invasion analysis; i.e., we consider the increase in frequency by selection of rare genetic mutations that control the growth strategy. There is a stable monorphic female brain size in the population when rare mutants of the growth strategy cannot invade the population; that is, such resident growth strategy is "uninvadable"31,32. We obtain an uninvadable growth strategy using evolutionary-invasion analysis for function-valued traits, since the growth strategy is a function of time (age). Because skill level ultimately depends on brain size due to energy conservation principles ${ }^{24}$, the evolution of brain size causes the evolution of skill level. Accordingly, a cooperating partner's skill level and the difficulty of competitive challenges are evolving environments, which constitutes the ultimate distinction between ecological and social challenges in our analysis. Such evolving environment implements the notion that sociality can yield evolutionary arms races in cognition as proposed by social hypotheses ${ }^{8-10}$.

Energy extraction efficiency. An important quantity in the model is the individual's energyextraction efficiency (EEE), defined as the rate of energy extraction divided by the rate of energy extraction if the individual is maximally successful at energy extraction. We model the 
individual's EEE as a function of her skill level and that of cooperating or competing peers. To do this, we consider two mathematical functions commonly used in contest models: a "power competence" function that allows for strongly-decelerating EEE as the individual gains skills when she is young, and an "exponential competence" function that allows for weaker deceleration (Fig. 2b and Extended Data Fig. 1c). We also let the skills of cooperating partners interact in an additive, multiplicative, or "submultiplicative" (geometric mean) way (the geometric mean is a good descriptor of the average skill in the pair if peers have disparate skill levels). Additionally, we assume that if a sufficiently young individual fails to overcome a challenge, then she can extract energy from an environment facilitated by her mother.

Parameters. The model has: 4 basic parameters, collectively denoted by $\mathbf{P}$, that specify the proportion of each social challenge, and whose effects we study here; 13 further parameters, collectively denoted by $\mathbf{Q}$, that measure the brain's and other tissues' metabolic costs, brain's and other tissues' size at birth, and demography, and for which empirical estimates are available; and a final 9 parameters, collectively denoted by $\mathbf{R}$, that measure skill metabolic costs, maternal provisioning, mutation size, and how skill level affects energy extraction, and for which we use reasonable values given the available data (Fig. 2c; Supplementary Information section 4). For example, R-parameters include the metabolic cost of memory and the values we use for this (in megajoules per year per terabyte) fall within an empiricallyestimated range for resting energy consumption for stored motor patterns in cerebellum Purkinje cells in rats ${ }^{33}$. The exact values used for $\mathbf{R}$ are chosen within such reasonable ranges as they yield a high ontogenetic fit between predicted and observed body and brain mass in H. sapiens when there are only ecological challenges (i.e., $P_{1}=1$; Extended Data Fig. 3g,h). This approach is a reasonable starting point given that the fundamental constraint for a large 
brain is thought to be the metabolic costs of brain, which are incorporated in the estimated $\mathbf{Q}$ parameters. The values chosen for the $\mathbf{R}$-parameters mean that the difficulty of ecological challenges is high but not exceedingly so, memory is metabolically expensive (although in the low end of the empirically-estimated range), and skills are moderately-effective at overcoming the challenges. Using such $\mathbf{Q}$ and $\mathbf{R}$-parameter values, it was previously shown that ecological challenges alone can generate adult brain and body sizes of ancient human scale: of late H. erectus scale with strongly-decelerating EEE and of Neanderthal scale with weaklydecelerating $\mathrm{EEE}^{24}$. Here we use the same $\mathbf{Q}$ and $\mathbf{R}$ parameter values and study the effects of the social-challenge parameters $\mathbf{P}$.

Key equations. We assume that the population is large and mostly constituted by individuals with a resident growth strategy and by vanishingly rare individuals with a mutant growth strategy. At age $t$, a focal mutant individual has a mass of tissue $i$ (for $i \in\{\mathrm{b}, \mathrm{r}, \mathrm{s}\}$ for brain, reproductive, and somatic) of $x_{i}(t)$ (in kilograms) and a skill level of $x_{\mathrm{k}}(t)$ (in terabytes). The growth rate of tissue $i \in\{\mathrm{b}, \mathrm{r}, \mathrm{s}\}$ is

$$
\dot{x}_{i}(t)=\frac{1}{E_{i}} u_{i}(t)\left[B_{\text {rest }}(t)-\sum_{i \in\{\mathrm{b}, \mathrm{r}, \mathrm{s}\}} B_{i} x_{i}(t)\right],
$$

where $\dot{x}_{i}(t)$ denotes the derivative of $x_{i}(t)$ with respect to $t$. The term in square brackets is the growth-metabolic rate $\left[B_{\text {syn }}(t)\right]$, which equals the resting metabolic rate, $B_{\text {rest }}(t)$, minus the maintenance metabolic rate, $\sum_{i \in\{\mathrm{b}, \mathrm{r}, \mathrm{s}\}} B_{i} x_{i}(t)$. The metabolic cost of producing (respectively, maintaining) a mass unit of tissue $i$ is $E_{i}$ (respectively, $B_{i}$ ). The growth strategy is the fraction of the growth-metabolic rate due to the production of each tissue throughout life, and for the mutant it is denoted by $u_{i}(t)$ for all $t$ and all $i \in\{\mathrm{b}, \mathrm{r}, \mathrm{s}\}$ (or $u$ for short). We let the growth strategy be the evolving trait. In turn, the mutant skill growth rate is

$$
\dot{x}_{\mathrm{k}}(t)=\frac{1}{E_{\mathrm{k}}}\left[s_{\mathrm{k}} B_{\mathrm{rest}, \mathrm{b}}(t)-B_{\mathrm{k}} x_{\mathrm{k}}(t)\right]
$$


The brain metabolic rate is $B_{\mathrm{rest}, \mathrm{b}}(t)$, and the metabolic cost of gaining (respectively, maintaining) a skill unit is $E_{\mathrm{k}}$ (respectively, $B_{\mathrm{k}}$ ). The fraction of brain metabolic rate allocated to energy-extraction skills is $s_{\mathrm{k}}$. Resting metabolic rate is a power function of body mass, $x_{\mathrm{B}}(t)$, and a function of EEE, which we denote by $e\left(x_{\mathrm{k}}(t), y_{\mathrm{k}}(t)\right)$ :

$$
B_{\text {rest }}(t)=K e\left(x_{\mathrm{k}}(t), y_{\mathrm{k}}(t)\right) x_{\mathrm{B}}^{\beta}(t)
$$

where $y_{\mathrm{k}}(t)$ is the skill level at age $t$ of a resident individual. The brain metabolic rate is the sum of brain's maintenance and growth metabolic rates:

$$
B_{\text {rest }, \mathrm{b}}(t)=B_{\mathrm{b}} x_{\mathrm{b}}(t)+E_{\mathrm{b}} \dot{x}_{\mathrm{b}}(t) .
$$

An uninvadable growth strategy $u_{i}^{*}(t)$ for all $t$ and all $i \in\{\mathrm{b}, \mathrm{r}, \mathrm{s}\}\left(\right.$ or $\left.\mathrm{u}^{*}\right)$ is a best response to itself (similar to a Nash equilibrium) regarding the lifetime number of offspring it yields ${ }^{31,32}$ (see Evolutionary differential game below). We denote the tissue mass and skill level resulting from an uninvadable growth strategy as $x_{i}^{*}(t)$ for all $t$ and all $i \in\{\mathrm{b}, \mathrm{r}, \mathrm{s}, \mathrm{k}\}$ (or $\mathbb{x}^{*}$ for short).

Switching times. With the parameter values we use, the uninvadable growth strategies typically produce a life history with four critical ages where the growth strategy changes suddenly (called switching times in optimal control terminology): the age of brain growth onset $t_{\mathrm{b} 0}$, which is when allocation to brain growth starts; the age of brain growth arrest $t_{\mathrm{b}}$, when allocation to brain growth stops; the age at maturity $t_{\mathrm{m}}$, when allocation to growth of reproductive tissue starts; and the age at adulthood $t_{\mathrm{a}}$, when allocation to growth of nonreproductive tissues stops. These four ages are an output, not parameters, of the model.

Asymptotic skill level. In adulthood (i.e., after $t_{\mathrm{a}}$ ), brain growth is absent and when memory is expensive enough skill growth asymptotically ceases $^{24}$. Specifically, $\dot{x}_{\mathrm{b}}^{*}(t)=0$ for $t \geq t_{\mathrm{a}}$ and 
$\dot{x}_{\mathrm{k}}^{*}(t)$ tends to 0 as $t$ tends to $T$, where $T$ is the age of menopause. Substituting this and Eq. (4) into Eq. (2) yields the asymptotic skill level

$$
\hat{x}_{\mathrm{k}}=s_{\mathrm{k}} \frac{B_{\mathrm{b}}}{B_{\mathrm{k}}} x_{\mathrm{b}}^{*}\left(t_{\mathrm{a}}\right) .
$$

Equations for energy extraction efficiency. In Supplementary Information section 2.1 we show that EEE can be written as

$$
e\left(x_{\mathrm{k}}(t), y_{\mathrm{k}}(t)\right)=\sum_{j=1}^{4} P_{j}\left[\frac{c_{j}}{c_{j}+d_{j}}+\frac{d_{j}}{c_{j}+d_{j}} \varphi\right] .
$$

The term in square brackets (denoted by $e_{j}$ ) gives the energy extraction efficiency when facing a challenge of type $j$ and is composed of two terms. The first term is the proportion of time the individual succeeds at the challenge, and the second term is the proportion of time the individual fails at the challenge but extracts energy from maternal provisioning. The individual's competence at a $j$-challenge is $c_{j}$ and in general depends on her own and on her social partners' skill level. The difficulty of a $j$-challenge is $d_{j}$ and in general depends on the social and non-social environment (i.e., on the social partners' skill level and the constant environment). Because the mutant is rare, her social partners are residents. The energy extraction efficiency from maternal provisioning is $\varphi$, which decreases with age.

Using our assumption of domain-general skills, we let the competence function be independent of the challenge type, $c_{j}\left(G_{j}\left(x_{\mathrm{k}}, y_{\mathrm{k}}\right)\right)=c\left(G_{j}\left(x_{\mathrm{k}}, y_{\mathrm{k}}\right)\right)$, where $G_{j}\left(x_{\mathrm{k}}, y_{\mathrm{k}}\right)$ is a production function describing how the skills of the cooperating partners interact (e.g., in an additive, multiplicative, or submultiplicative way). We consider two forms for the competence function:

$$
c\left(G_{j}\left(x_{\mathrm{k}}, y_{\mathrm{k}}\right)\right)= \begin{cases}G_{j}^{\gamma}\left(x_{\mathrm{k}}, y_{\mathrm{k}}\right) & \text { with power competence } \\ \exp \left[G_{j}\left(x_{\mathrm{k}}, y_{\mathrm{k}}\right)\right]^{\gamma} & \text { with exponential competence, }\end{cases}
$$


where $\gamma$ measures the effectiveness (decidability) of skills at the challenge. The production function $G_{j}\left(x_{\mathrm{k}}, y_{\mathrm{k}}\right)$ is

$$
G_{j}\left(x_{\mathrm{k}}, y_{\mathrm{k}}\right)= \begin{cases}x_{\mathrm{k}} & \text { for } j \in\{1,3\} \\ x_{\mathrm{k}}+y_{\mathrm{k}} & \text { for } j \in\{2,4\} \text { with additive cooperation } \\ x_{\mathrm{k}} y_{\mathrm{k}} & \text { for } j \in\{2,4\} \text { with multiplicative cooperation } \\ \sqrt{x_{\mathrm{k}} y_{\mathrm{k}}} & \text { for } j \in\{2,4\} \text { with sub-multiplicative cooperation }\end{cases}
$$

The difficulty $d_{j}$ of a challenge depends on the challenge type. For an ecological or a cooperative challenge, the challenge difficulty is $\alpha$ which depends on the "ecological" environment, which we assume constant (this assumption can be relaxed in future extensions; Supplementary Information section 9). In turn, the difficulty of a competitive challenge depends on the skill level of the individual's competitors. Since the mutant is rare, a mutant's competitors are residents, so the difficulty of a competitive challenge is the competence of the resident, $c\left(G_{j}\left(y_{\mathrm{k}}, y_{\mathrm{k}}\right)\right)$. In general, the difficulty of a type- $j$ challenge is

$$
d_{j}\left(y_{\mathrm{k}}\right)= \begin{cases}\alpha & \text { for } j \in\{1,2\} \\ c\left(G_{j}\left(y_{\mathrm{k}}, y_{\mathrm{k}}\right)\right) & \text { for } j \in\{3,4\} .\end{cases}
$$

We let the energy extraction efficiency from maternal provisioning when the individual is of age $t$ be

$$
\varphi(t)=\varphi_{0} \exp \left(-\varphi_{\mathrm{r}} t\right)
$$

where $\varphi_{0}$ is the energy extraction efficiency from maternal provisioning at birth and $\varphi_{\mathrm{r}}$ measures the rate of decrease of maternal provisioning. The resulting equations for $e_{j}$ for all cases considered are in the Supplementary Information section 2.3. 
Evolutionary differential game. Let $R_{0}(\mathfrak{u}, \mathbb{w})$ be the expected lifetime number of offspring of a mutant with growth strategy ou when the resident growth strategy is $\mathbb{v}$. We assume that the population is kept at a constant size due to density dependence competition through fertility rather than survival. Hence, an uninvadable growth strategy $u^{*}$ maximizes the mutant's lifetime number of offspring when the mutant is resident ${ }^{34}$. That is, the mutant satisfies

$$
\mathrm{u}^{*} \in \underset{\mathrm{u} \in \mathbb{U}}{\arg \max _{0}} R_{0}\left(\mathfrak{u}, \mathrm{u}^{*}\right)
$$

where $\mathbb{U}$ is the set of feasible growth strategies. Assuming that the mortality rate $\mu$ is constant (which can be relaxed in future extensions; Supplementary Information section 9) and that reproductive tissue is narrowly defined so it is not involved in offspring maintenance (e.g., defined as preovulatory ovarian follicles), the mutant's lifetime number of offspring when $\mathbb{v}$ is resident is

$$
R_{0}(\mathfrak{u}, \mathbb{v}) \propto \int_{0}^{T} \exp (-\mu t) x_{\mathrm{r}}(t) \mathrm{d} t
$$

Eq. (11) poses a differential game problem: it is a "game" between mutant and resident because the mutant's payoff $\left[R_{0}(\mathfrak{u}, \mathbb{v})\right]$ depends on the resident strategy, it is "differential" because it depends on differential equations [Eqs. (1) and (2)], and it is "evolutionary" rather than a typical differential game because only the mutant's payoff is maximized rather than both the mutant and resident's payoffs. Because problem (11) involves maximization and its solutions are functions $\left(\mathrm{u}^{*}, \mathbb{x}^{*}\right)$, this maximization poses an optimal control problem. We solve problem (11) numerically by iterating optimal control problems until convergence to a point where the mutant and resident strategies are indistinguishable to a chosen extent. To do so, we use the software GPOPS ${ }^{35}$.

Figure specifications. For Fig. 3a-b, plots are around only ecological challenges; that is, for a given plot, the remaining two $P_{j}$ 's are set to zero. The arrows in Fig. 3c describe the quali- 
tative effect determined in Fig. 3a-b of increasing the proportion of a social challenge as the proportion of ecological challenge decreases, or of increasing the proportion of ecological challenges as the proportion of cooperative challenges decreases. The patterns in Fig. 3a-c also hold around the best-fitting $\mathbf{P}^{*}$ for $H$. sapiens; that is, when for a given plot, the remaining two $P_{j}$ 's are set to the values of $\mathbf{P}^{*}$ (Extended Data Figs. $4 \mathrm{~b}, \mathrm{c}$ ). "Missing" dots in Fig. $3 \mathrm{~d}$ are $P_{j}$-combinations that did not converge to an uninvadable growth strategy [e.g., due to cycling solutions, suggesting possible evolutionary branching (female dimorphism) in brain size] or that were unreachable from lack of convergence of nearby runs (Supplementary Information section 5). For Fig. 3a-e, cooperation is submultiplicative, and for Fig. 3d-e, competence is exponential (see Extended Data Fig. 4 for all cases).

Fig. 4a shows the hominin species for which we find a near-perfect adult fit (i.e., where the best adult fit is greater than the chosen threshold of $-D\left(\tau_{\mathrm{a}}\right)=-0.05$; Extended Data Figs. 6-8). For Fig. 4a, cooperation is submultiplicative (resp. additive) for weakly- (resp. strongly-) decelerating EEE. In Fig. 4b, dots are the values for an average $H$. sapiens female as reported by ref. ${ }^{21}$. The resulting life periods in Fig. $4 \mathrm{~b}$ are defined as "childhood", where there is no allocation to production of reproductive tissue; "adolescence", where there is allocation to production of somatic and reproductive tissues; and "adulthood", where there is only allocation to production of reproductive tissue. The energy extraction efficiency from maternal provisioning at birth (part of the $\mathbf{R}$ parameters) in Fig. $4 \mathrm{~b}$ is slightly smaller than its benchmark value to improve ontogenetic fit further without affecting adult fit (ontogenetic fit is $-\mathrm{E}[D(\tau)]=-0.22$ using $\varphi_{0}^{*}=0.5$ rather than -0.33 using the benchmark $\varphi_{0}=0.6$; Supplementary Information sections 6 and 8). 
Code availability. Code that supports the findings of this study is available in Zenodo with the identifier https://doi.org/10.5281/zenodo.1197479.

Data availability. Data of predicted brain size, body size, and skill level for the various challenge combinations as generated by this study and as used for Figs. 3 and 4 and Extended Data Figs. 4 and 6-8 have been deposited in Zenodo with the identifier https://doi.org/10.5281/zenodo.1197479. Complete numerical solutions including growth strategies across the parameter sweep totalling 200 GB of data are available from the corresponding author upon request.

31. Lehmann, L., Mullon, C., Akçay, E. \& Van Cleve, J. Invasion fitness, inclusive fitness, and reproductive numbers in heterogeneous populations. Evolution 70, 1689-1702 (2016).

32. Lion, S. Theoretical approaches in evolutionary ecology: environmental feedback as a unifying perspective. Am. Nat. 191, 21-44 (2018).

33. Howarth, C., Peppiatt-Wildman, C. M. \& Attwell, D. The energy use associated with neural computation in the cerebellum. J. Cereb. Blood Flow Metab. 30, 403-414 (2010).

34. Mylius, S. D. \& Diekmann, O. On evolutionarily stable life histories, optimization and the need to be specific about density dependence. Oikos 74, 218-224 (1995).

35. Patterson, M. A. \& Rao, A. V. GPOPS-II: A MATLAB Software for solving multiple-phase optimal control problems using hp-adaptive Gaussian quadrature collocation methods and sparse nonlinear programming. ACM Trans. Math. Softw. 41, 1-37 (2014).

36. Schniter, E., Gurven, M., Kaplan, H. S., Wilcox, N. T. \& Hooper, P. L. Skill ontogeny among Tsimane forager-horticulturalists. Am. J. Phys. Anthrop. 158, 3-18 (2015). 
a

Energy extraction efficiency, $e$

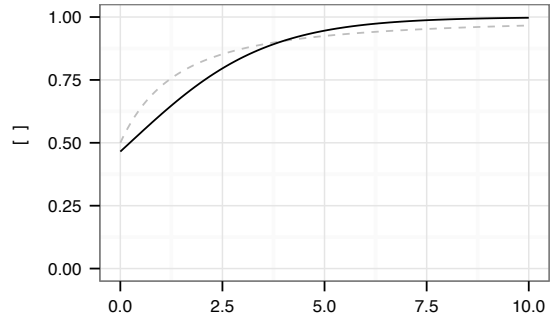

b

Speed of energy extraction efficiency, $\partial e / \partial x_{k}$

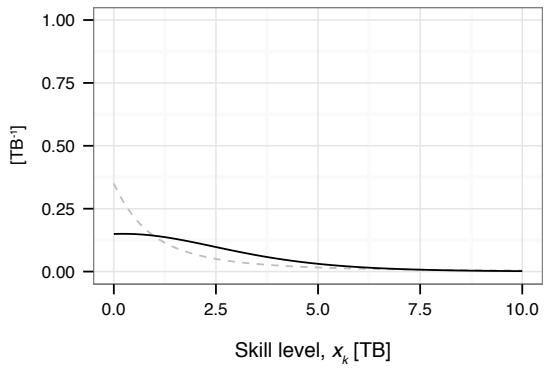

C energy extraction efficiency, $\partial^{2} e / \partial x_{k}^{2}$

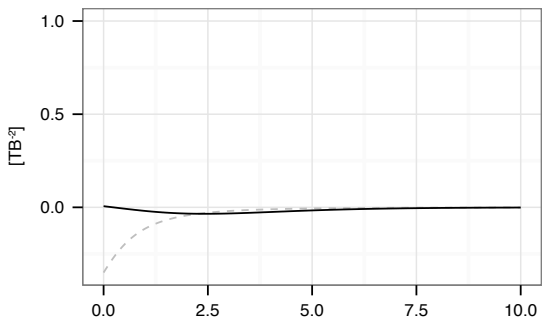

Power competence

Exponential competence 
Extended Data Figure 1: Shape of EEE vs skill. a-c, Plots of energy extraction efficiency $e$, its speed, and acceleration with respect to skill level under power competence (PC) and exponential competence (EC) with only ecological challenges (i.e., $P_{1}=1$ ) for the parameter values used. For comparison, the curves for PC are displaced to the left by 1 unit because skill level at birth is 1 for PC but 0 for EC. a-b, $e$ and its speed at birth and during young ages are smaller for EC than for PC, but $\mathbf{c}$, the acceleration in $e$ at birth and at young ages is larger for EC than for PC. 

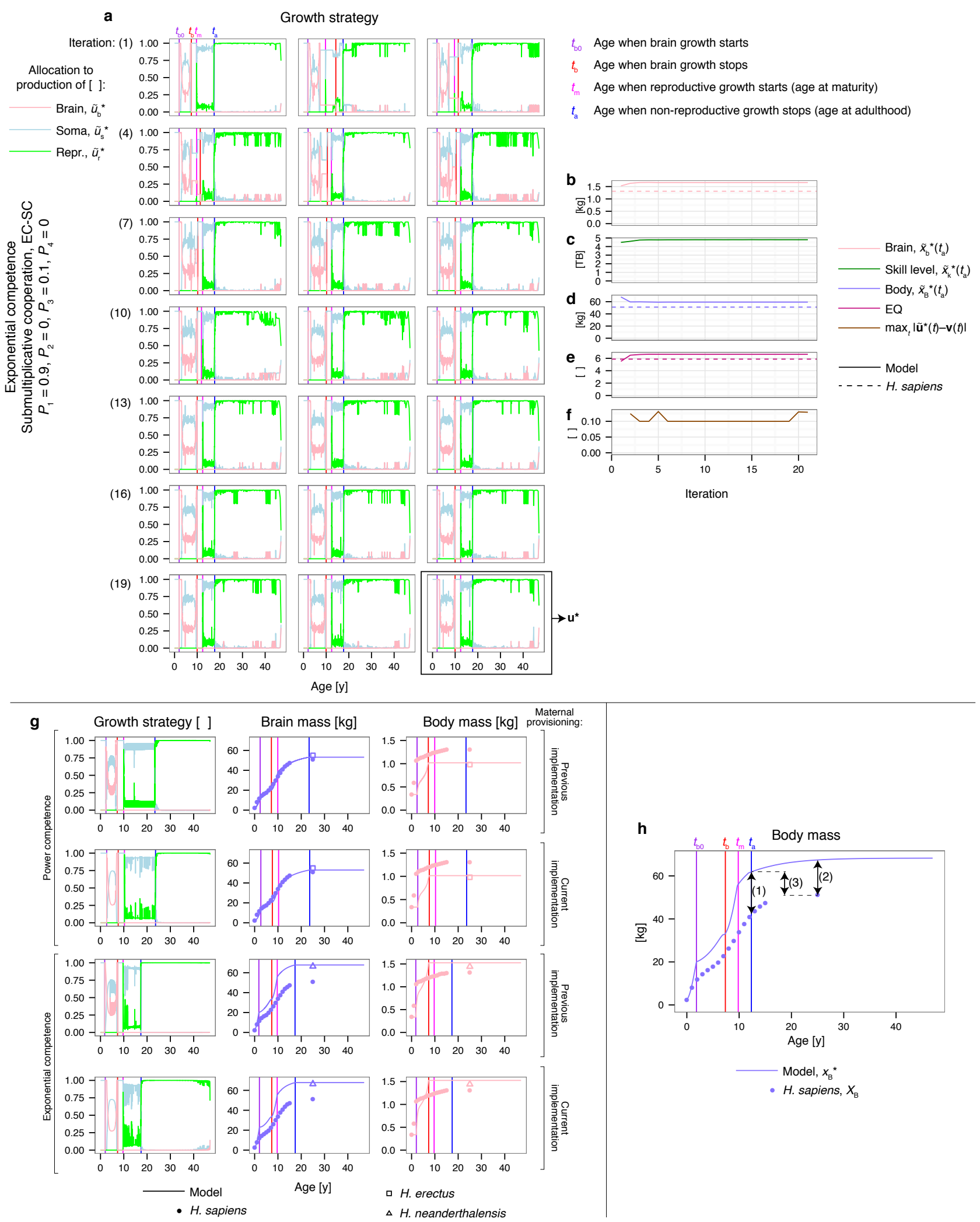
Extended Data Figure 2: Method implementation. a, Typical result with convergence to an uninvadable growth strategy. For the $(i)$-th iteration, the growth strategy shown is the resident (v) whose best response $\left(\widetilde{\mathbf{u}}^{*}\right)$ is shown next, which is the resident of the $(i+1)$-st iteration. Convergence to a best response to itself $\left(\mathbf{u}^{*}\right)$ was declared visually (in a at iteration 21). b-f, Reporting variables across the iterations in a. b-e, Resulting adult body mass, brain mass, skill level, and EQ through iterations. These values tend to converge more quickly than the growth strategy (a). f, Rather than visually declaring convergence, convergence should ideally be declared when the difference between mutant and resident is below a chosen threhold. However, numerical jittering prevents the use of this criterion. For example, $\mathbf{f}$ shows the maximum of $\left|\widetilde{\mathbf{u}}^{*}(t)-\mathbf{v}(t)\right|$ across $t$ for each iteration in $\mathbf{a}$. Without numerical jittering, this maximum should decrease as the growth strategy approaches a best response to itself. Yet, numerical jittering causes this maximum to be at least equal to the maximum mutation size $\delta=0.1$. The maximum of $\left|\widetilde{\mathbf{u}}^{*}(t)-\mathbf{v}(t)\right|$ is occasionally greater than $\delta$ because $\widetilde{\mathbf{u}}^{*}$ and $\mathbf{v}$ have different partitions over $t$. Thus, to calculate $\left|\widetilde{\mathbf{u}}^{*}(t)-\mathbf{v}(t)\right|$, for each $t$ in the $t$-partitioning of $\widetilde{\mathbf{u}}^{*}$, we find the closest $t$ in the $t$-partitioning of $\mathbf{v}$ and calculate the difference at these relatively close times; this may occasionally cause the difference to be larger than $\delta$ when strategies change suddenly with $t$. Alternative measures of convergence were similarly inadequate (e.g., $\left.\sum_{t}\left|\widetilde{\mathbf{u}}^{*}(t)-\mathbf{v}(t)\right|\right)$. g, We implement maternal provisioning differently than before ${ }^{24}$ to incorporate it when there are social challenges. The difference yields no detectable difference in predicted brain and body mass with only ecological challenges after slightly adjusting the energy extraction efficiency from maternal provisioning of a newborn $\left(\varphi_{0}\right)$ : before ${ }^{24}, \varphi_{0}=0.6$ for power and $\varphi_{0}=0.8$ for exponential competence; here, $\varphi_{0}=0.4$ for power and $\varphi_{0}=0.6$ for exponential competence. $\mathbf{h}$, Three ways to measure adult fit: (1) at the predicted age of adulthood $\left[x_{\mathrm{B}}\left(t_{\mathrm{a}}\right)-X_{\mathrm{B}}\left(t_{\mathrm{a}}\right)\right]$; (2) at the observed age of adulthood $\left[x_{\mathrm{B}}\left(\tau_{\mathrm{a}}\right)-X_{\mathrm{B}}\left(\tau_{\mathrm{a}}\right)\right]$; and (3) at the predicted age of adulthood for the prediction and at the observed age of adulthood for the observation $\left[x_{\mathrm{B}}\left(t_{\mathrm{a}}\right)-X_{\mathrm{B}}\left(\tau_{\mathrm{a}}\right)\right]$. We use option (2). 
a
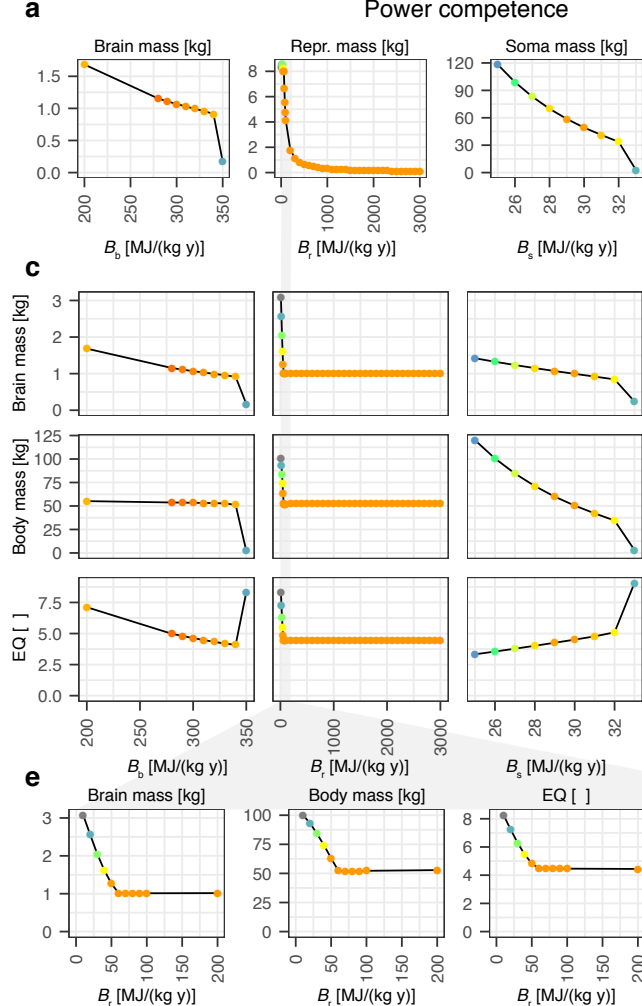

$B_{\mathrm{s}}[\mathrm{MJ} /(\mathrm{kg} \mathrm{y})]$
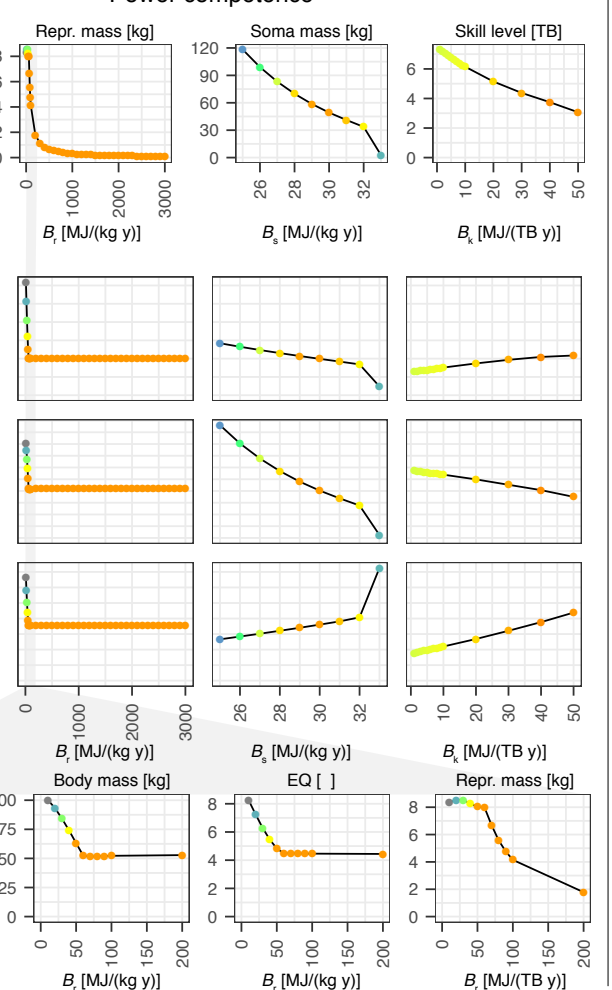

b
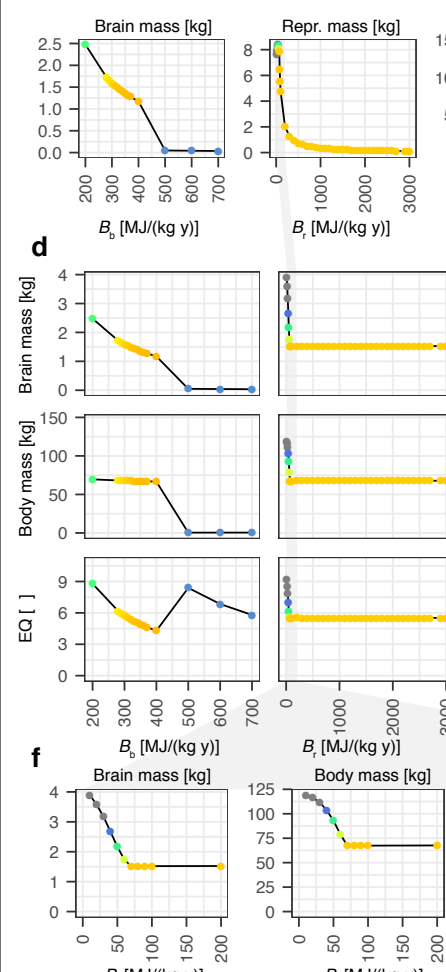

Exponential competence
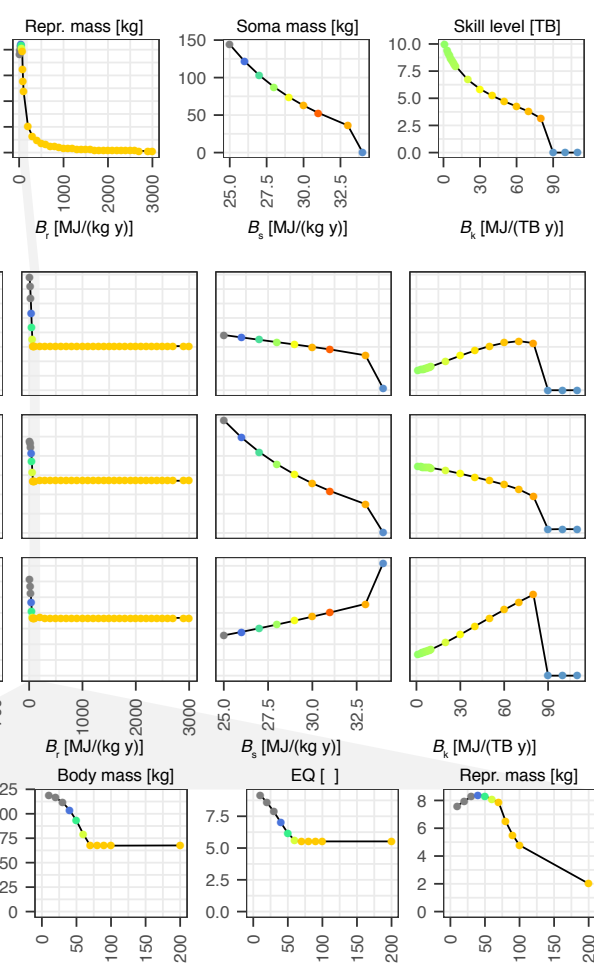

Repr. mass $[\mathrm{kg}]$

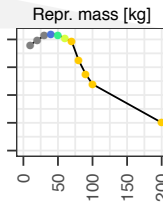

B [MJ/(TB y)]

$\begin{array}{lllll} & -1.5 & -1.0 & -0.5 & 0.0\end{array}$

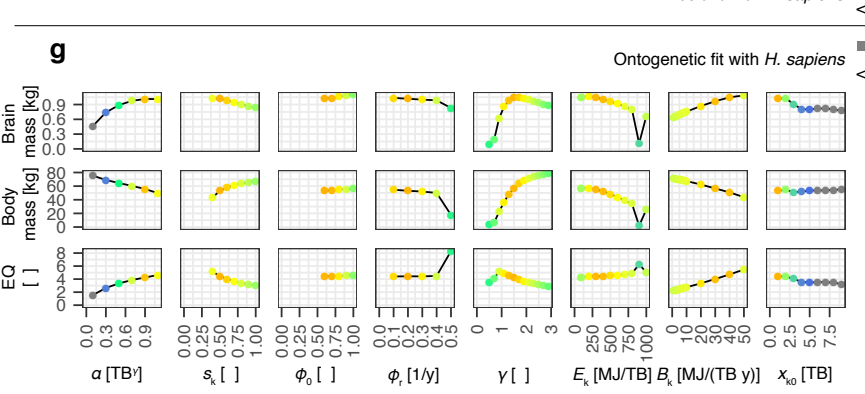

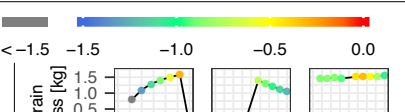
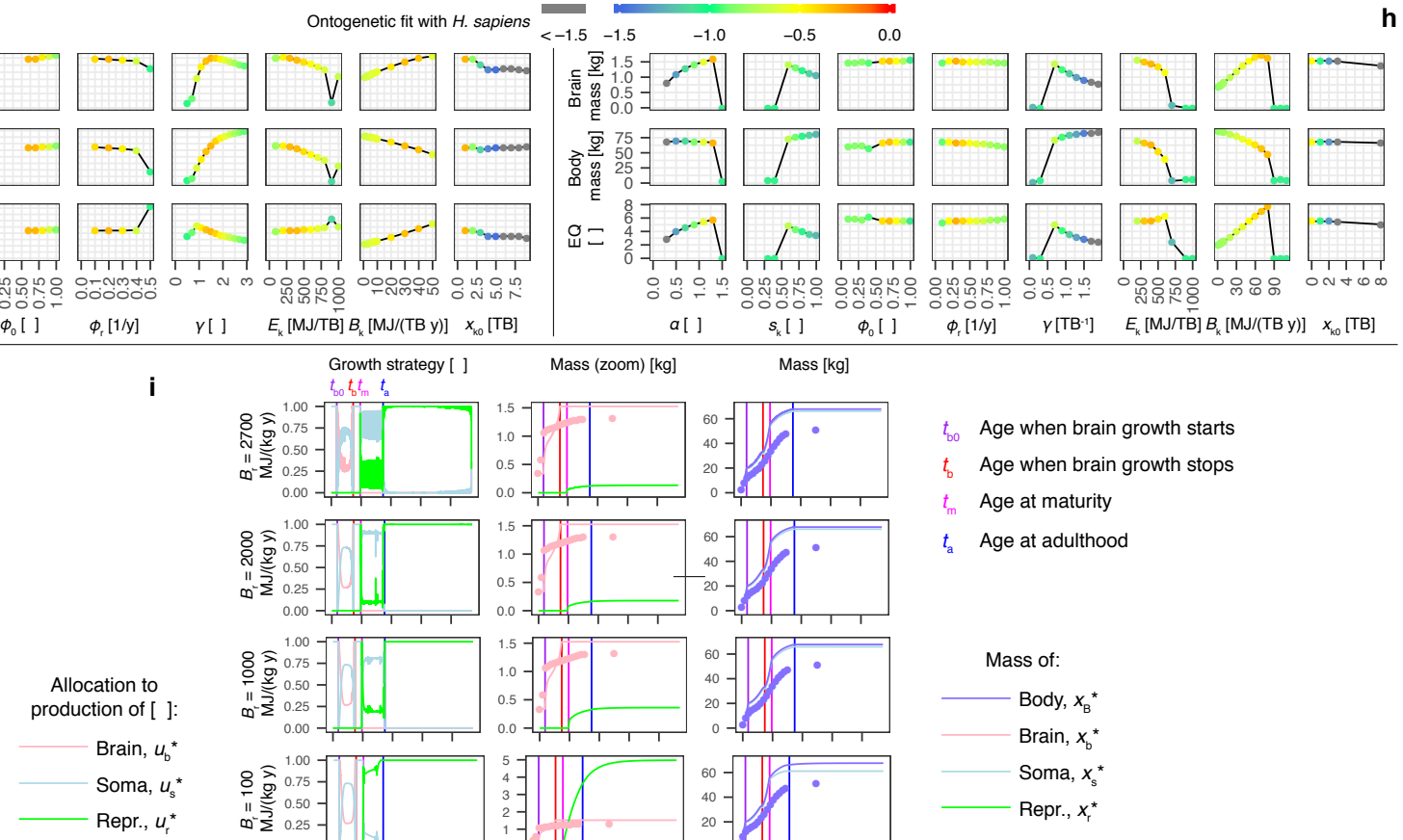

$t_{\mathrm{b} 0}$ Age when brain growth starts

$t_{\mathrm{b}}$ Age when brain growth stops

$t_{\mathrm{m}}$ Age at maturity

$t_{\mathrm{a}} \quad$ Age at adulthood
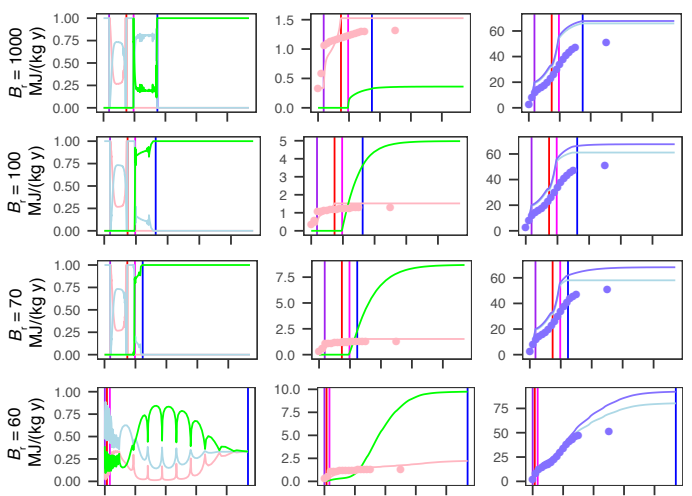

Mass of:

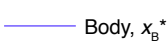

Brain, $x_{\mathrm{b}}^{*}$

Soma, $x_{\mathrm{s}}^{*}$

Repr., $x_{\mathrm{r}}^{*}$

Model, $\boldsymbol{x}_{i}^{\star}$

- H. sapiens, $X_{\mathrm{B}}$ 
Extended Data Figure 3: Effects of $\mathbf{Q}$ and $\mathbf{R}$ parameters. a-b, Effects of maintenance costs $\left(B_{i}\right)$ on the corresponding tissue mass or skill level. Each $B_{i}$ tends to decrease the value $x_{i}^{*}\left(\tau_{\mathrm{a}}\right)$ of the corresponding $i$, but not necessarily of the other $i$ [see (c-d)]. c-d, Effect of $B_{i}$ on adult brain mass, body mass, and EQ. With power competence (c), when $B_{\mathrm{b}}=310$ and $340 \mathrm{MJ} /(\mathrm{kg} \mathrm{y})$, the predicted adult brain mass is $x_{\mathrm{b}}^{*}\left(\tau_{\mathrm{a}}\right)=1.0298$ and $0.9133 \mathrm{~kg}$, respectively. With exponential competence (c), when $B_{\mathrm{b}}=310,340$, and $370 \mathrm{MJ} /(\mathrm{kg} \mathrm{y})$, the predicted adult brain mass is $x_{\mathrm{b}}^{*}\left(\tau_{\mathrm{a}}\right)=1.542,1.3973$, and $1.2767 \mathrm{~kg}$, respectively. e-f, Effects of $B_{\mathrm{r}}$ when $B_{\mathrm{r}}$ is small. When $B_{\mathrm{r}}$ varies between 70 and $2700 \mathrm{MJ} /\left(\mathrm{kg}\right.$ y), $B_{\mathrm{r}}$ has no detectable effect on adult brain mass and EQ. g-h, Ontogenetic fit with $H$. sapiens around the used values for each of the $\mathbf{R}$ parameters (except $\delta$ ). The ontogenetic fit is approximately maximized around the benchmark values chosen previously ${ }^{24}$ which are also used here (except for $\varphi_{0}$ given our improved implementation of $\varphi$ ). i, Effect of $B_{\mathrm{r}}$ on the predicted life history with exponential competence. For $\mathbf{i}$, in the left column, from top to bottom row, as $B_{\mathrm{r}}$ decreases, the allocation to the growth of reproductive tissue during adolescence increases $\left(u_{\mathrm{r}}^{*}\right.$ between $t_{\mathrm{m}}$ and $t_{\mathrm{a}}$ ) and adolescence shortens. For $\mathbf{i}$, in the central column, the increased allocation to the growth of reproductive tissue increases the mass of reproductive tissue, but brain mass does not change with $B_{\mathrm{r}}$ for $B_{\mathrm{r}} \geq 70 \mathrm{MJ} /(\mathrm{kg} \mathrm{y})$. For $\mathbf{i}$, in the right column, as the mass of reproductive tissue increases, body mass increases slightly, which is more noticeable for $B_{\mathrm{r}} \leq 100$ $\mathrm{MJ} /(\mathrm{kg} \mathrm{y})$. An exceedingly small $B_{\mathrm{r}}[<70 \mathrm{MJ} /(\mathrm{kg} \mathrm{y})]$ disrupts the predicted life history, which with $B_{\mathrm{r}}=60 \mathrm{MJ} /(\mathrm{kg}$ y) is severely different from that of $H$. sapiens (e.g., there is brain growth late in life and reproductive growth from birth). Similar results arise for still smaller $B_{\mathrm{r}}$. In a-i there are only ecological challenges and we use the previous ${ }^{24}$ definition of $\varphi$. 
a

Around ecological challenges
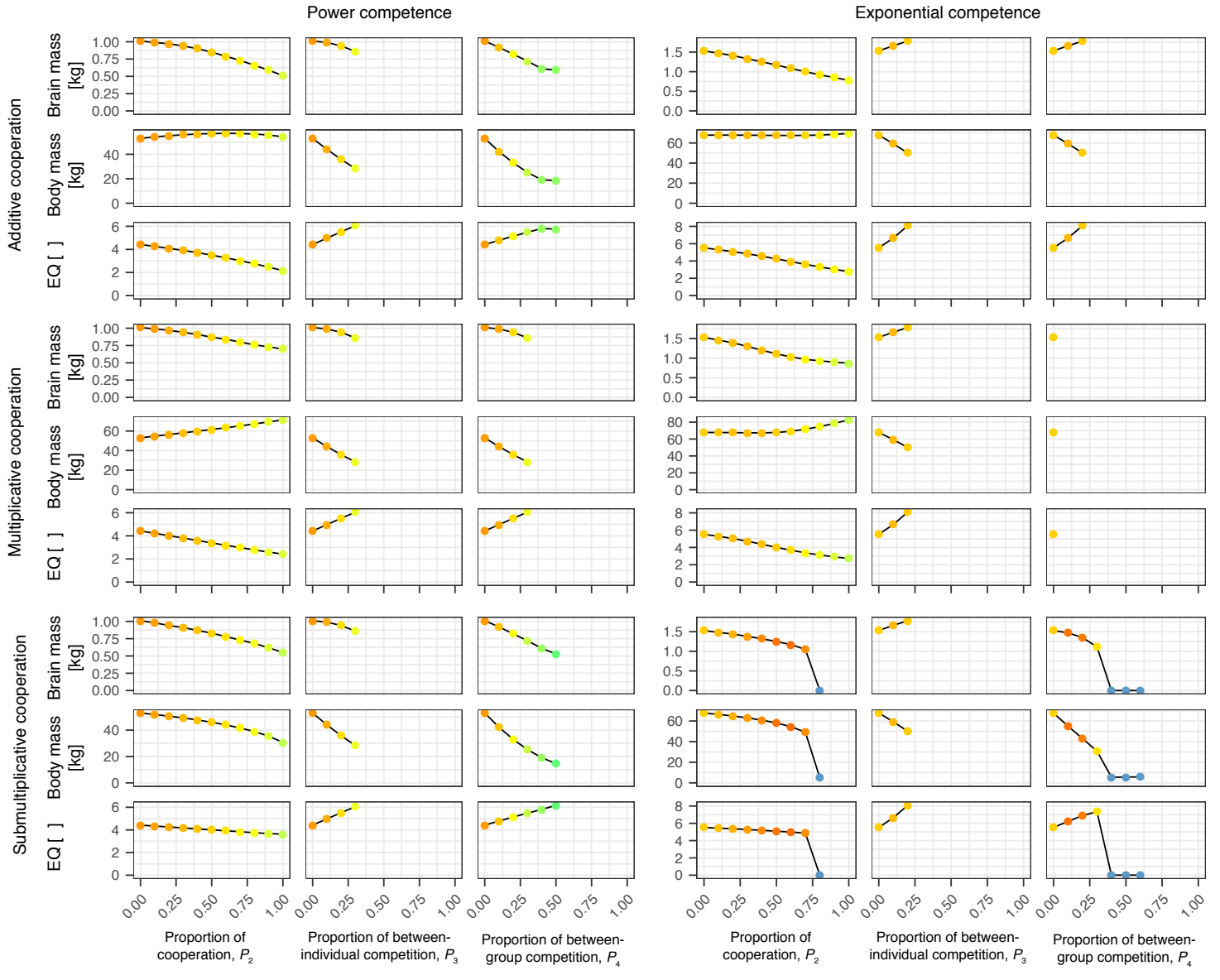

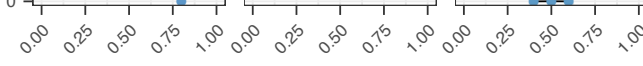

$\begin{array}{ccc}\begin{array}{c}\text { Proportion of } \\ \text { cooperation, } P_{2}\end{array} & \begin{array}{c}\text { Proportion of between- } \\ \text { individual competition, } P_{3}\end{array} & \begin{array}{c}\text { Proportion of between- } \\ \text { group competition, } P_{4}\end{array}\end{array}$

Around best fitting eco-social scenario
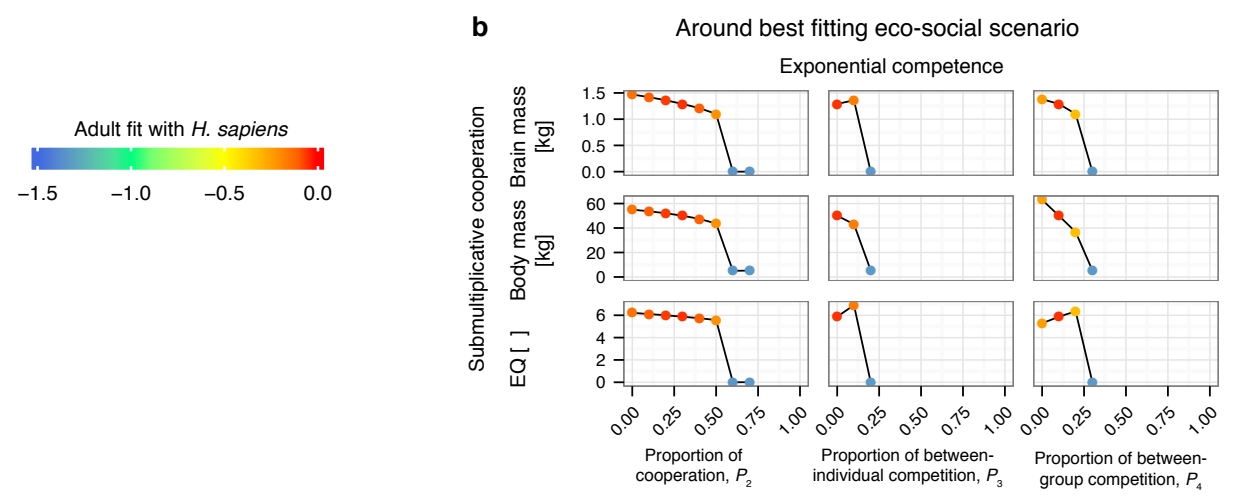

c

Around best-

fitting eco-

social scenario

\begin{tabular}{|c|c|c|c|c|c|c|c|c|c|c|c|c|c|c|c|c|c|c|c|c|c|}
\hline \multirow[b]{4}{*}{ Challenge type } & \multicolumn{18}{|c|}{ Around ecological challenges } & \multirow{3}{*}{\multicolumn{3}{|c|}{$\begin{array}{c}\frac{\text { social scenario }}{\text { EC }} \\
\text { SC }\end{array}$}} \\
\hline & \multicolumn{9}{|c|}{ Power competence } & \multicolumn{9}{|c|}{ Exponential competence } & & & \\
\hline & & $A C$ & & & MC & & & SC & & & $A C$ & & & MC & & & so & & & & \\
\hline & $\begin{array}{l}\frac{5}{\bar{\pi}} \\
\frac{0}{0}\end{array}$ & 중 & 욤 & 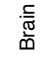 & $\begin{array}{l}\text { ते } \\
\text { வ }\end{array}$ & 品 & 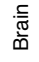 & 중 & 욤 & 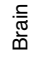 & $\begin{array}{l}\text { ते } \\
\text { வ }\end{array}$ & 욤 & $\frac{\frac{5}{\bar{\pi}}}{\frac{\pi}{0}}$ & ते & ơ & 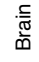 & 긍 & 品 & $\begin{array}{l}\frac{\complement}{\bar{N}} \\
\frac{\bar{\omega}}{2}\end{array}$ & 함 & 욤 \\
\hline $\begin{array}{l}\text { Ecological } \\
\text { (me vs nature) }\end{array}$ & & & & & & & & & & & & & & & & & & & & & \\
\hline $\begin{array}{l}\text { Cooperative } \\
\text { (us vs nature) }\end{array}$ & & & & & & & & & & & & & & & & & & & & & \\
\hline $\begin{array}{l}\text { Between-individ } \\
\text { competitive } \\
\text { (me vs you) }\end{array}$ & & & & & & & & & & & & & & & & & & & & & \\
\hline $\begin{array}{l}\text { Between-group } \\
\text { competitive } \\
\text { (us vs them) }\end{array}$ & & & & & & & & & & & & & & & & & & & & & \\
\hline
\end{tabular}


Extended Data Figure 4: Effects of challenge types on brain size. In a-b, outer rows are for the cooperation cases considered; outer columns are for the competence cases. $\mathbf{a}$ is around the pure ecological scenario (i.e., in a given plot for $P_{j}$ as $P_{1}$ decreases, the remaining two $P_{j}$ 's are set to zero). $\mathbf{b}$ is around the best fitting scenario for $H$. sapiens (i.e., in a given plot for $P_{j}$ as $P_{1}$ decreases, the remaining two $P_{j}$ 's are set to the best fitting $\mathbf{P}^{*}$ found in Fig. $3 \mathrm{~d}$ of the main text). c, Summary of the qualitative effects of challenge types on brain size. For social challenges, the direction of the arrows is taken from a-b. For ecological challenges, the direction of the arrow is taken from Extended Data Fig. 3g as the environmental difficulty $\alpha$ increases. A dash (-) indicates an approximately invariant relationship and a dot (·) indicates insufficient data points for identifying a relationship. The arrows in Fig. $3 c$ of the main text are taken from this summary, where for social challenges, the arrows are those of submultiplicative cooperation. AC: additive cooperation; MC: multiplicative cooperation; SC: submultiplicative cooperation; and EC: exponential competence. 
a

Exponential competence Submultiplicative cooperation, EC-SC

$P_{1}=0.4, P_{2}=0.4, P_{3}=0.2, P_{4}=0$

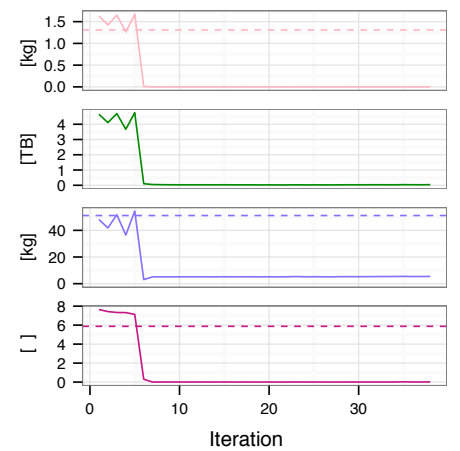

d

Exponential competence Submultiplicative cooperation, EC-SC $P_{1}=0.3, P_{2}=0.5, P_{3}=0.2, P_{4}=0$

$\left.\begin{array}{r}1.5- \\ 1.0- \\ \mathbb{\Xi}_{\Xi} \\ 0.5 \\ 0.0\end{array}\right]$

몬

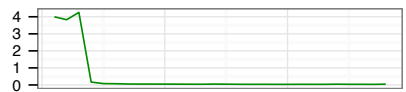

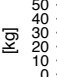

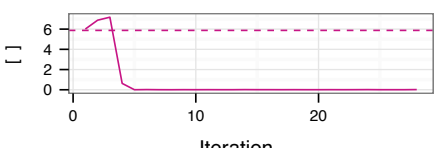

Power competence Submultiplicative cooperation, PC-SC $P_{1}=0.2, P_{2}=0.3, P_{3}=0.1, P_{4}=0.4$

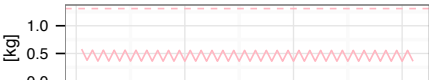

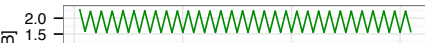
1.0
0.5

$\begin{array}{r}50 \\ 40 \\ \text { 实 } 30 \\ \hline\end{array}$

20
10
0

6 - manamanamanamadmamamak

工 $4-$

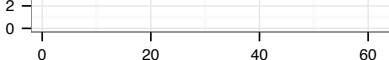

Iteration
C

Exponential competence Multiplicative cooperation, EC-MC $P_{1}=0.5, P_{2}=0.4, P_{3}=0, P_{4}=0.1$

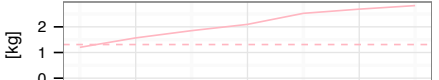

0

巴⿸尸 $\left.\begin{array}{l}8 \\ 6 \\ 4 \\ 2\end{array}\right]$

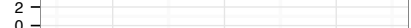

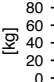

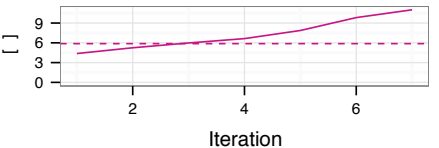

e Power competence

Submultiplicative cooperation, PC-AC $P_{1}=0.3, P_{2}=0.2, P_{3}=0, P_{4}=0.5$

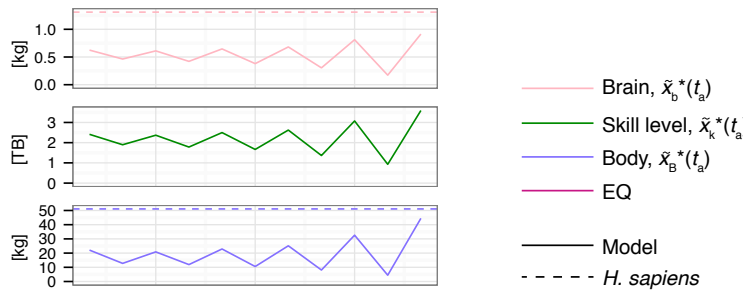


Extended Data Figure 5: Typical results when there is convergence to no brain growth or when there is no convergence to an uninvadable growth strategy. a-e, Adult values through iterations of best response to resident for cases of no brain growth or no convergence to an uninvadable strategy. a, Amplifying cycle leads to no brain growth. b, Stable cycle. c, Arms race that ends when the solver warns that the optimal control problem (OCP) may be infeasible. This suggests that the best response to the last iteration may involve a substantially different growth strategy, which is not allowed in the optimization as the best response is constrained to be sufficiently similar to that in the previous iteration. It is possible that such substantially different best response involves either no brain growth [e.g., as seen under purely ecological challenges when the environmental difficulty is exceedingly high $^{24}$ (Supplementary Information section 4.4)] or substantially more allocation to brain growth (which appears unlikely given the energetic constraints). d, A short arms race in EQ that leads to no brain growth. e, Amplifying cycle that ends when the solver warns that the OCP may be infeasible. 


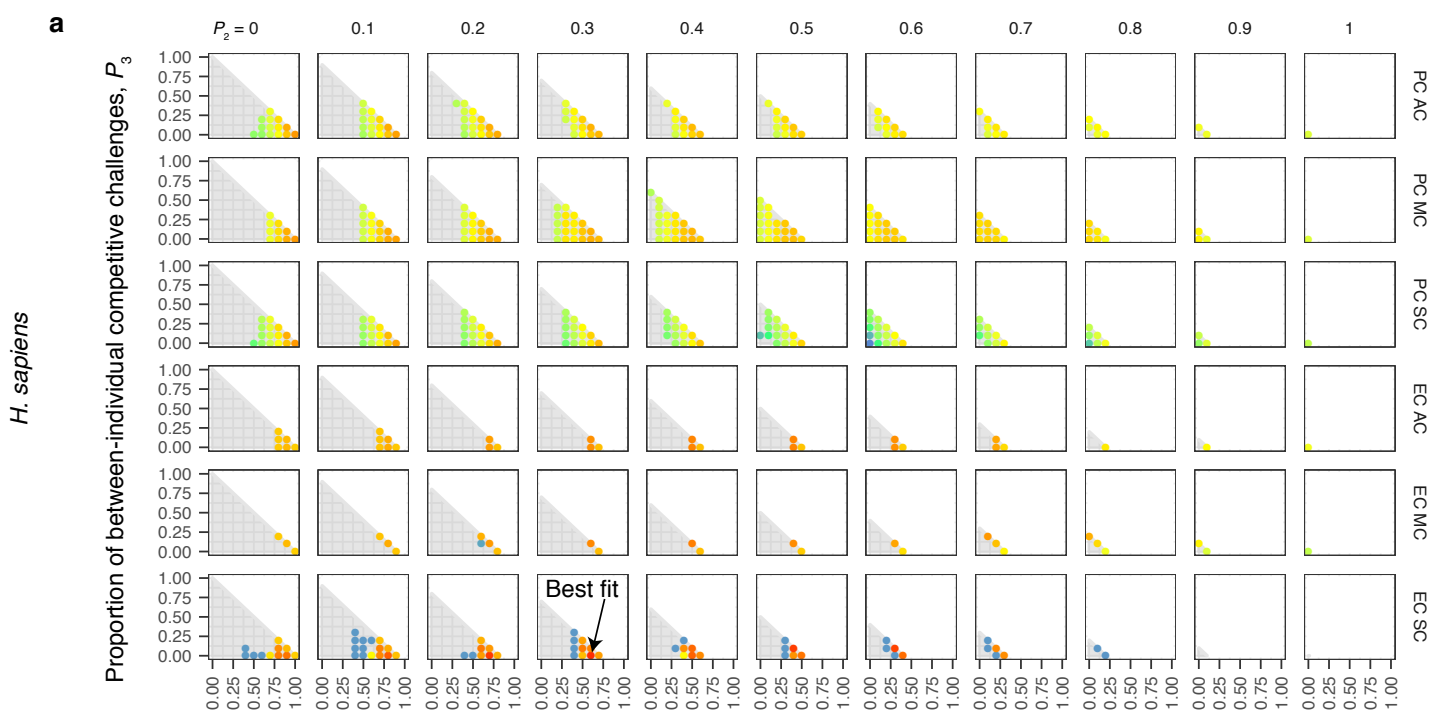
Proportion of ecological challenges, $P_{1}$

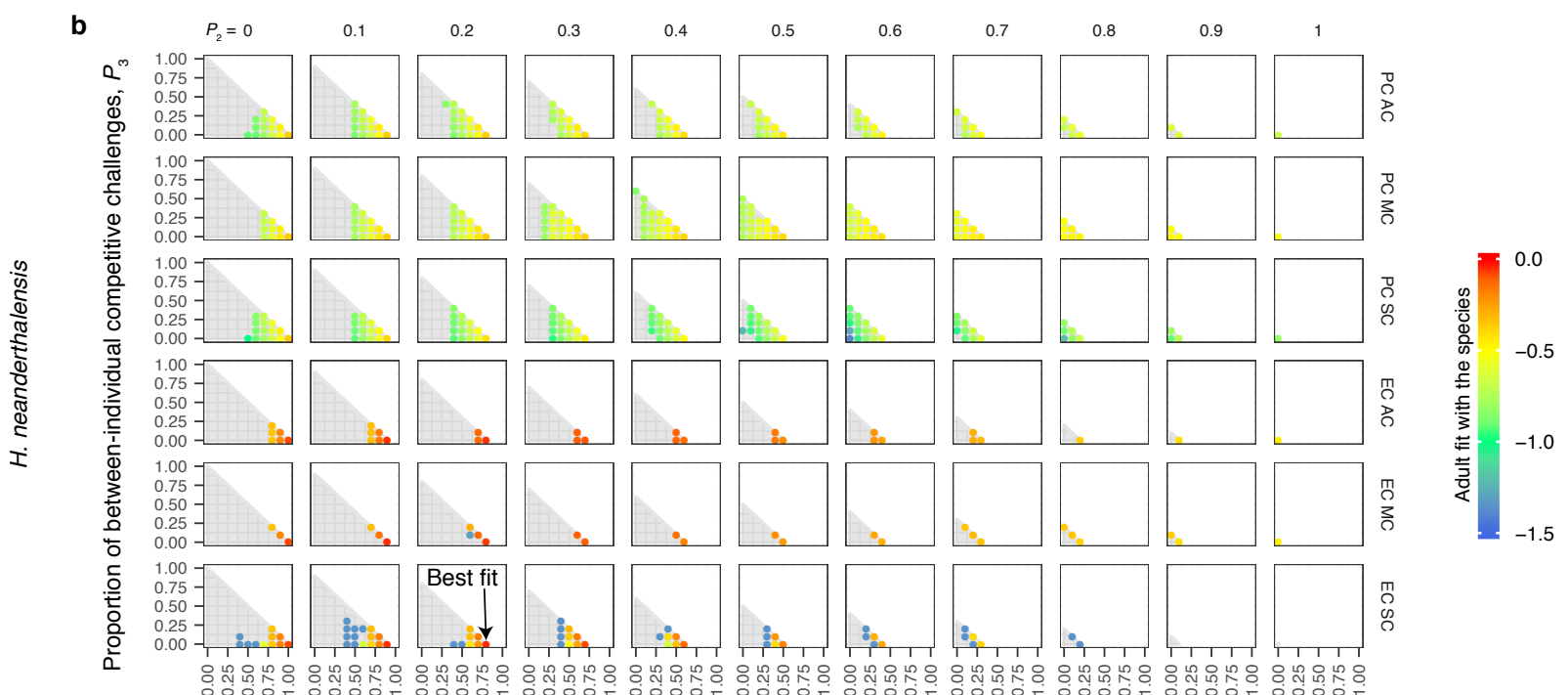
Proportion of ecological challenges, $P_{1}$

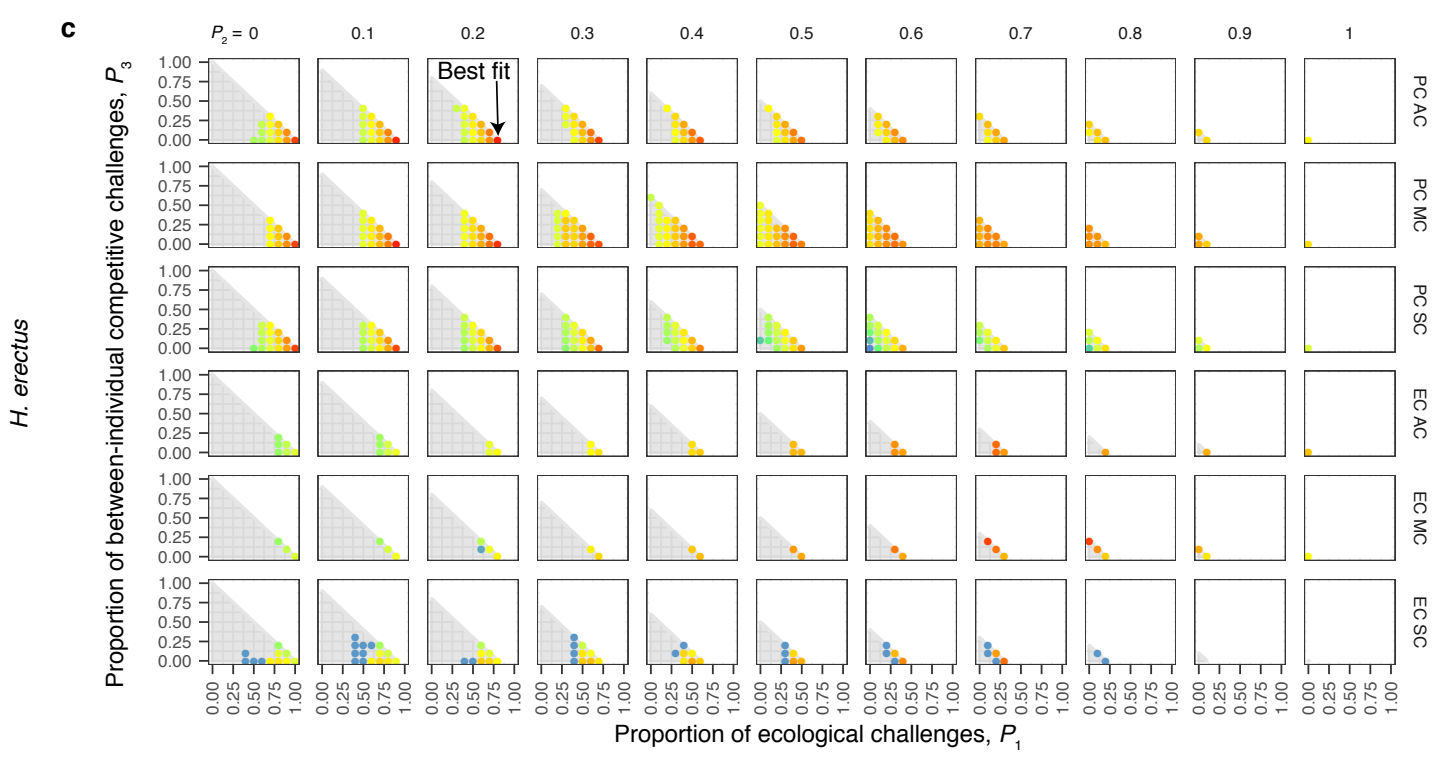


Extended Data Figure 6: Identification of best-fitting scenarios across hominins. Adult fit of predicted adult brain and body mass with those observed in a given species across parameter values for all cases considered. Each dot's colour gives the adult fit, $-D\left(\tau_{\mathrm{a}}\right)$, for the corresponding parameter combination and case. For a, H. sapiens, b, H. neanderthalensis, and c, $H$. erectus. 


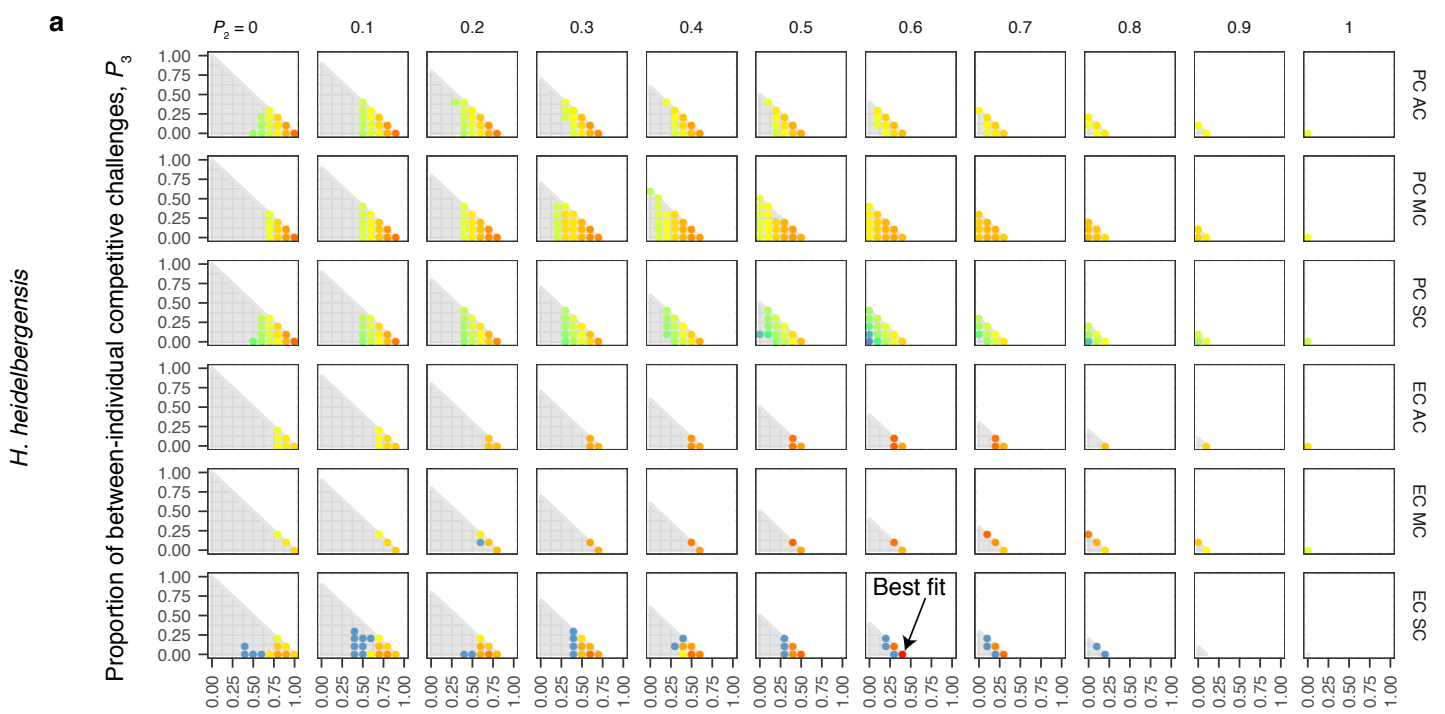

Proportion of ecological challenges, $P_{1}$

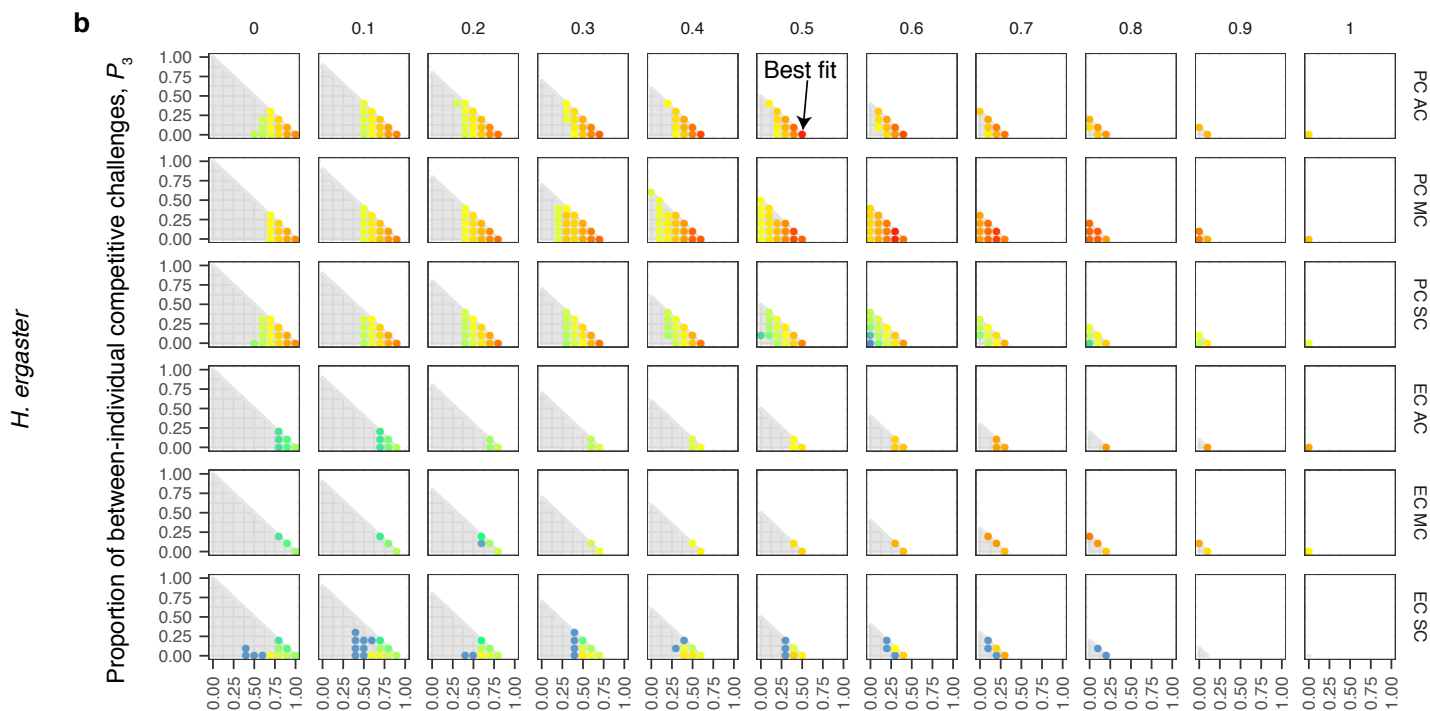
Proportion of ecological challenges, $P_{1}$

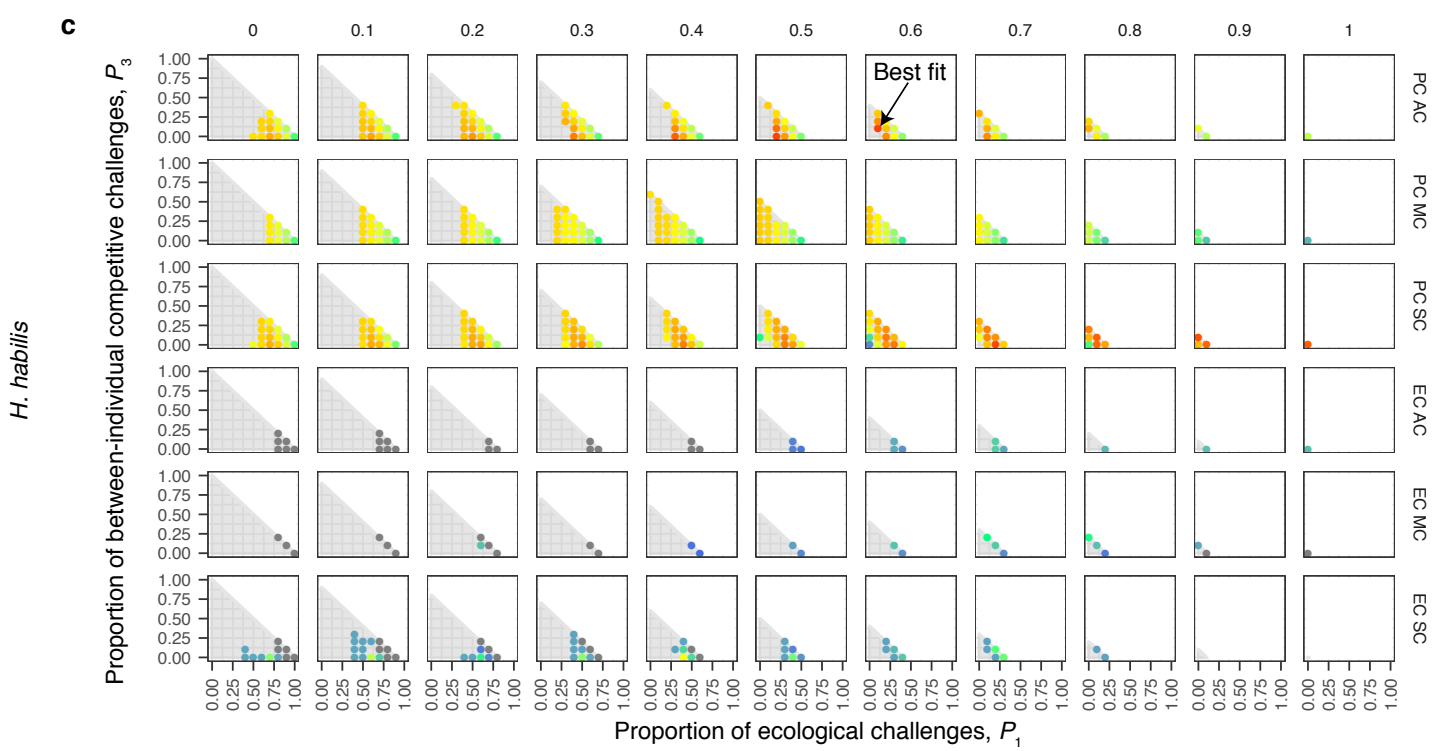


Extended Data Figure 7: Identification of best-fitting scenarios across hominins, continued. See legend of Extended Data Fig. 6 for details. For a, H. heidelbergensis, b, H. ergaster, and c, H. habilis. 


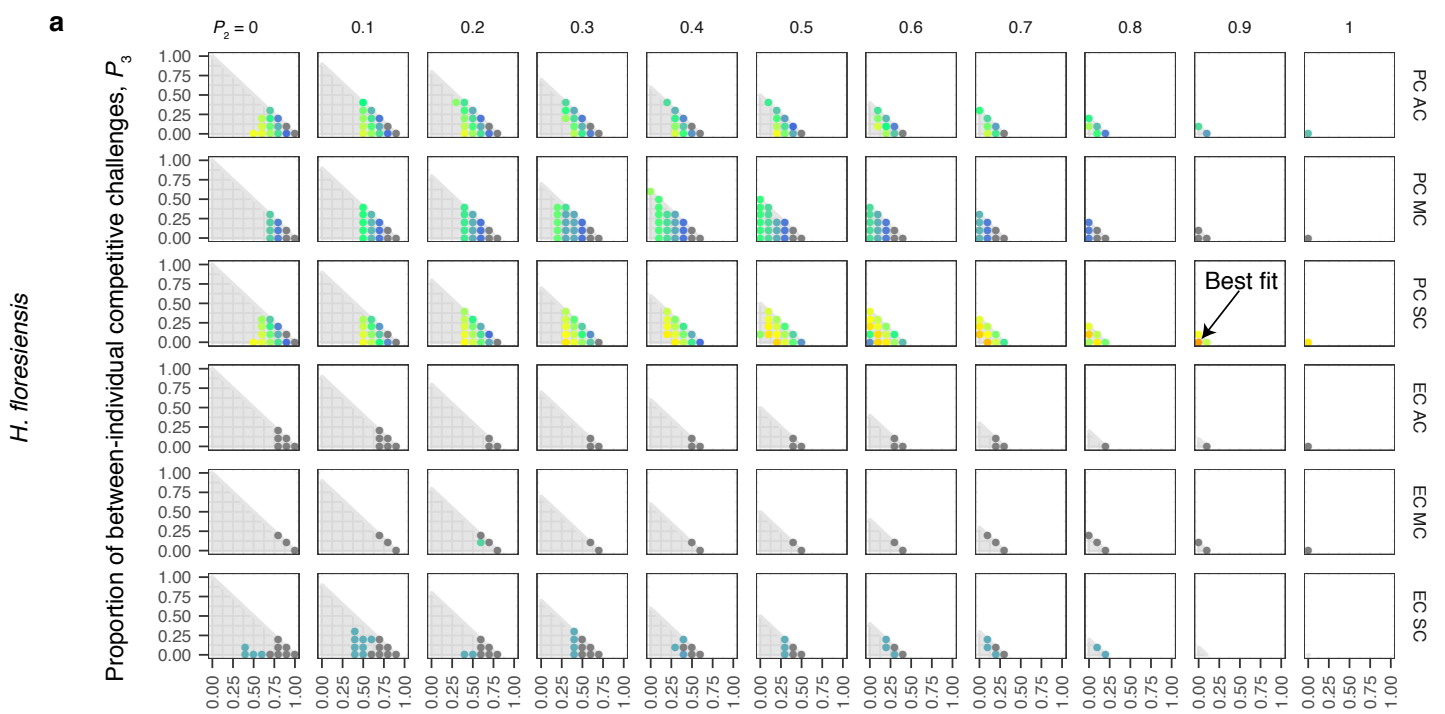
Proportion of ecological challenges, $P_{1}$

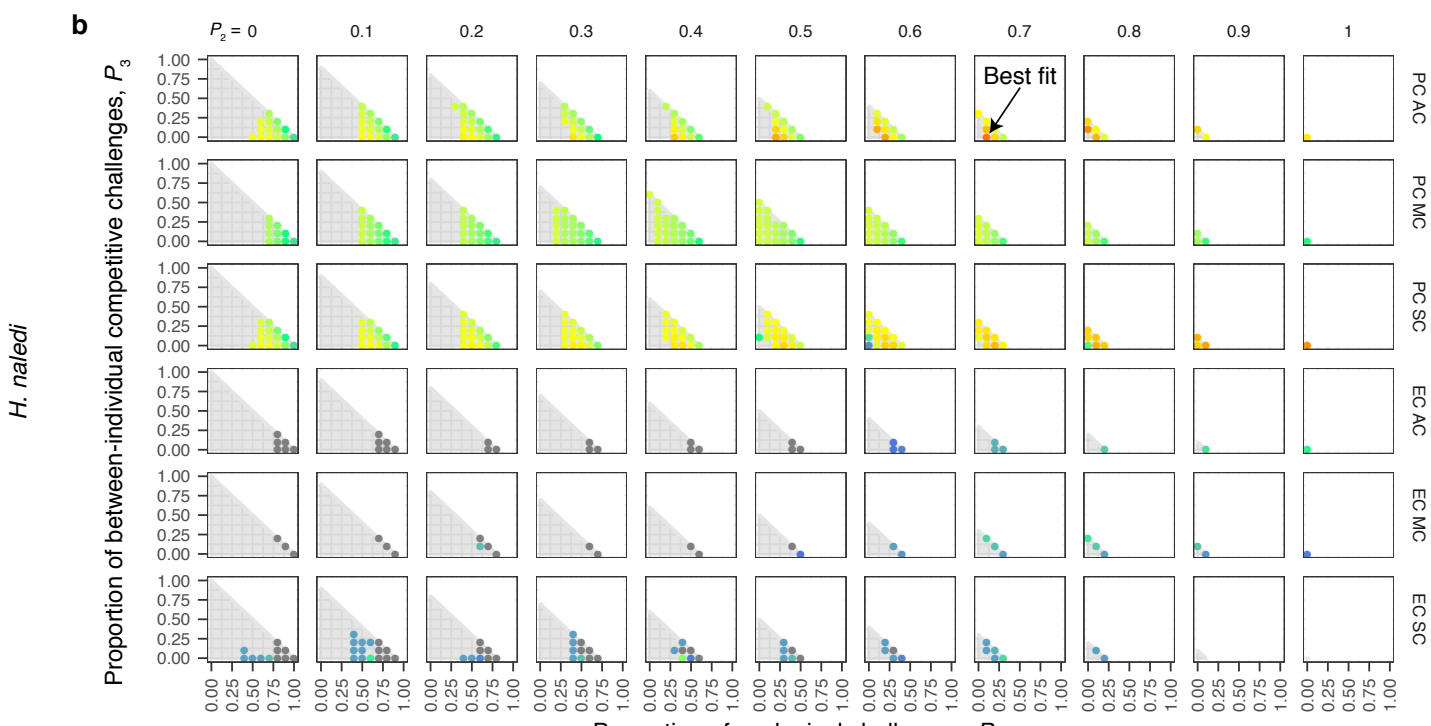
Proportion of ecological challenges, $P_{1}$

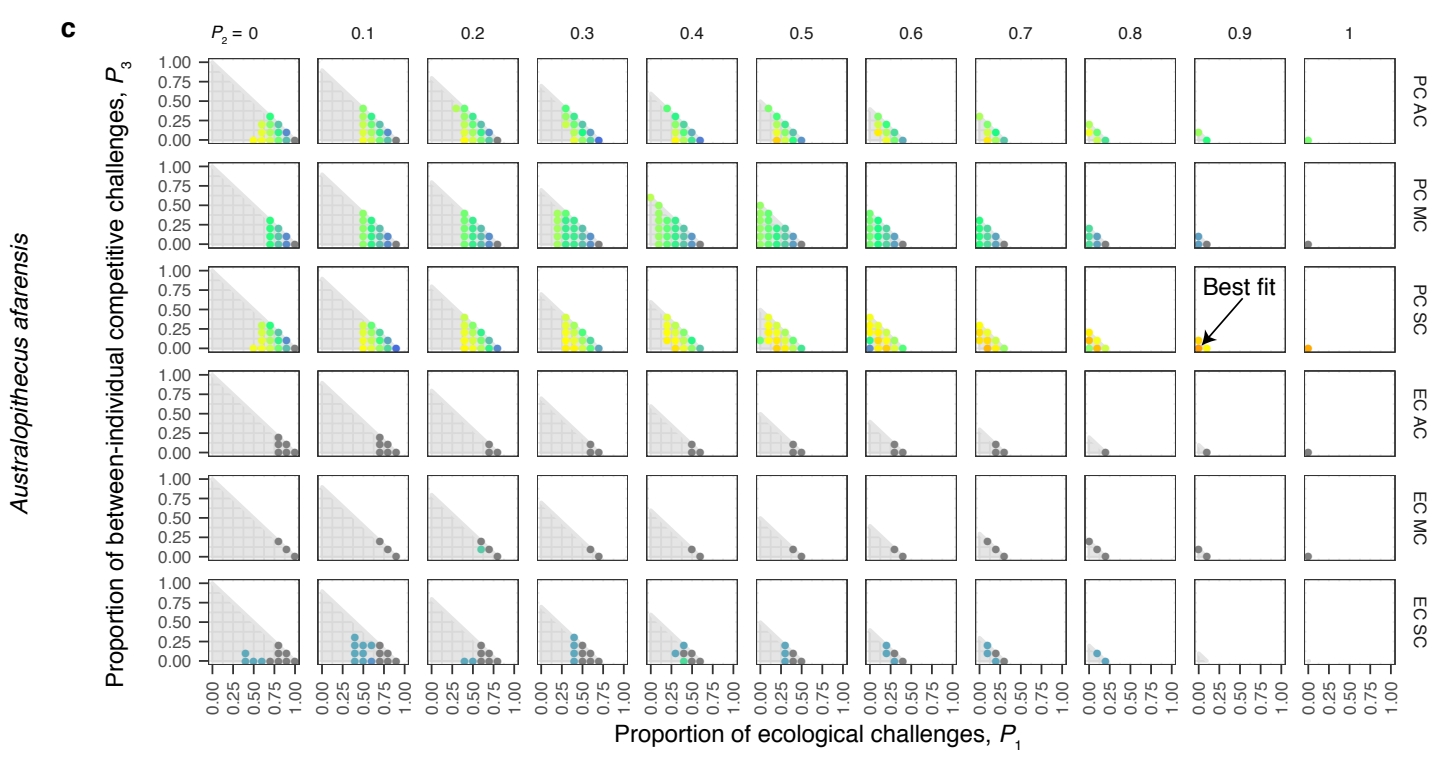


Extended Data Figure 8: Identification of best-fitting scenarios across hominins, continued. See legend of Extended Data Fig. 6 for details. For a, H. floresiensis, b, H. naledi, and c, Australopithecus afarensis. The best adult fit is $\mathbf{a},-0.24, \mathbf{b},-0.14$, and $\mathbf{c},-0.23$. 

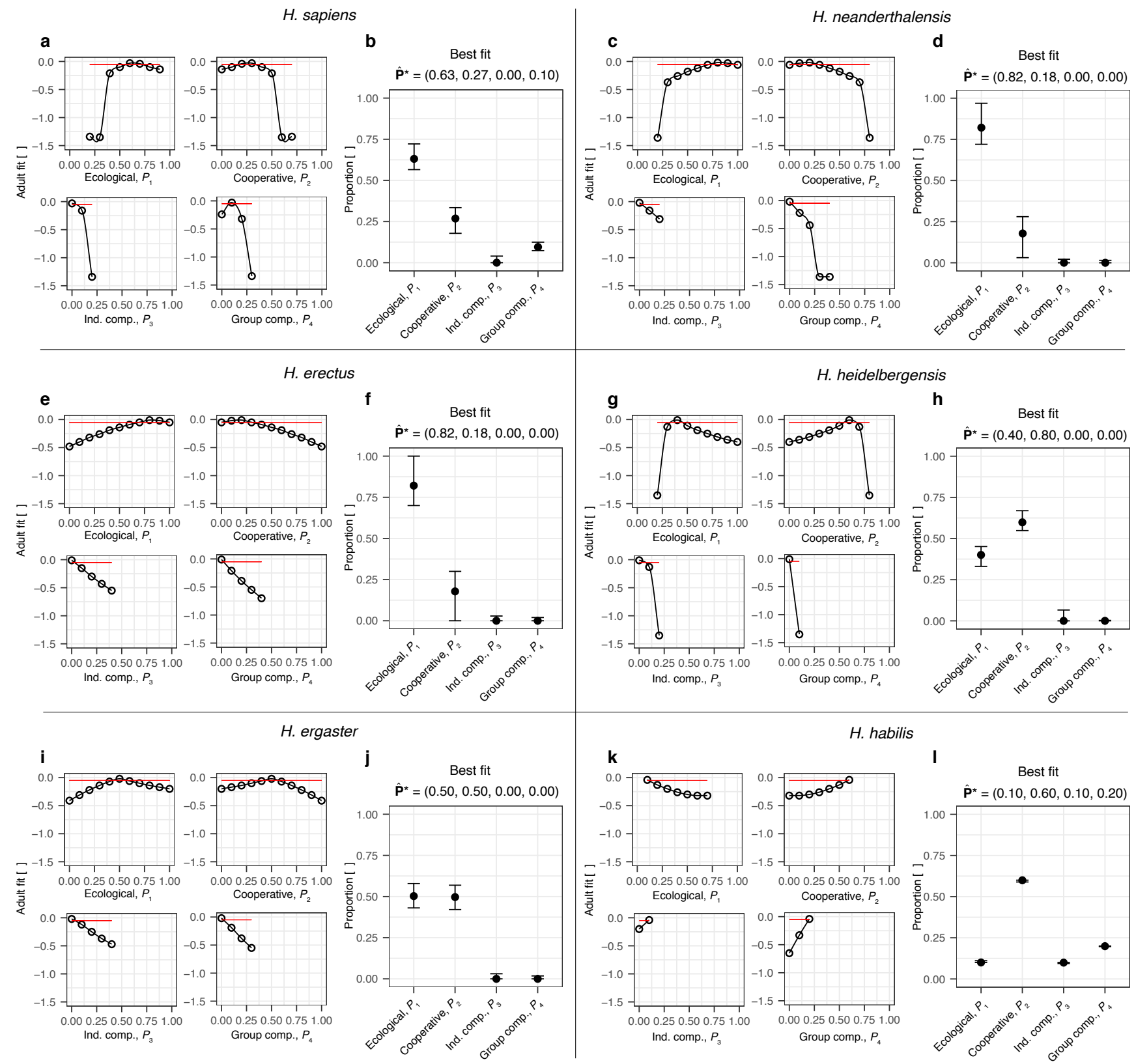
Extended Data Figure 9: High fit intervals for best fitting scenarios across hominins. Here we show high fit intervals around the best fitting scenarios across hominins having a best adult fit greater than -0.05 . a,c,e,g,i,k, For the top left plot, as $P_{1}$ increases, $P_{2}$ decreases, while for the remaining plots as $P_{2}, P_{3}$, and $P_{4}$ increase, $P_{1}$ decreases; for a given plot, the remaining $P_{j}$ are set to the corresponding $\mathbf{P}^{*}$ shown in Fig. 4 a in the main text (i.e., plots are around $\mathbf{P}^{*}$ ). The dots are the adult fit and the lines are interpolated values using a monotone Hermite spline (splinefun with method monoH. FC in R). The red line is $-D\left(\tau_{\mathrm{a}}\right)=-0.05$.

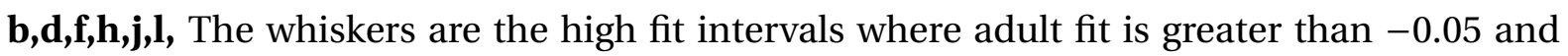
the dots are the estimated $\hat{\mathbf{P}}^{*}$ giving the best adult fit for the species in the interpolation. Competence and cooperation are as found in Extended Data Figs. 6 and 7. Note that for H. habilis the high fit intervals may be wider as the adult fit is increasing at the end of the values of $P_{2}, P_{3}$, and $P_{4}$ for which uninvadable growth strategies were obtained. 

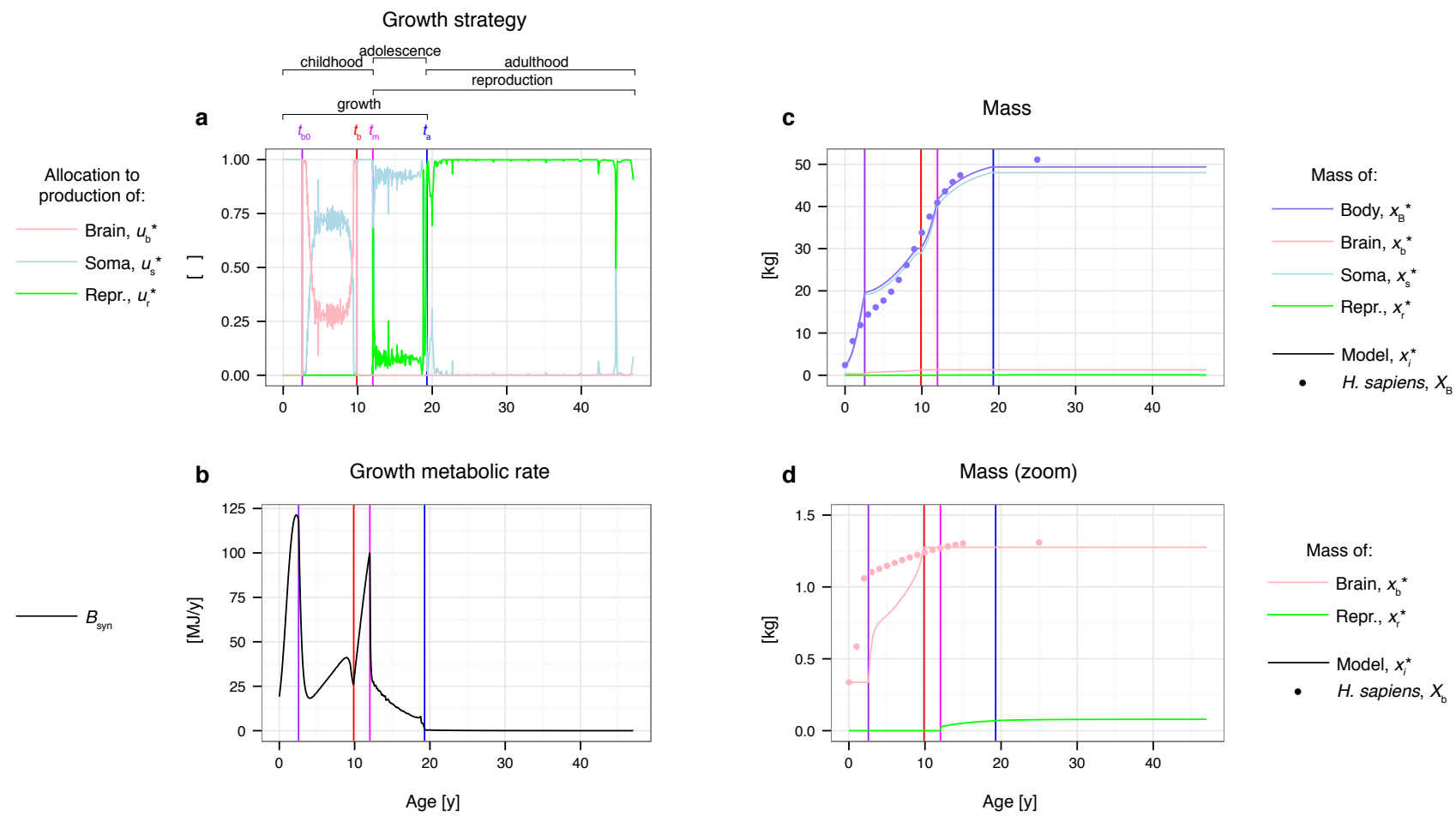

e

Skill level

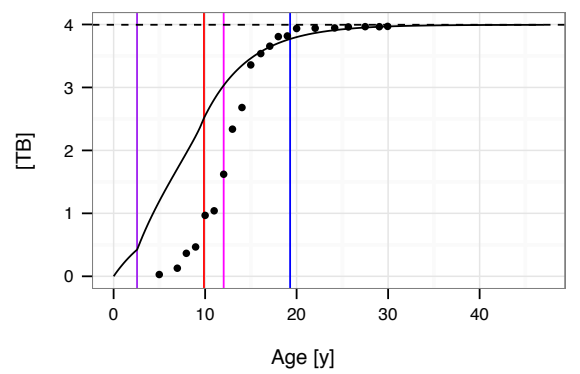

- Model, $x_{\mathrm{k}}{ }^{*}$

- - - Asymptotic skill level, $\hat{x}_{k}$ 
Extended Data Figure 10: Detailed life history resulting from the best-fitting scenario for H. sapiens. Plots correspond to Fig. $4 \mathrm{~b}$ in the main text and show a, the growth strategy generating the life history, $\mathbf{b}$, the resulting growth metabolic rate, $\mathbf{c}-\mathbf{d}$, the mass of all tissues, and e, the skill level. For comparison with the model's predicted skill level $x_{\mathrm{k}}^{*}$, the black dots in $\mathbf{e}$ are the observed cumulative distribution of self-reported acquisition ages of food production skills in female Tsimane horticulturalists ${ }^{36}$ multiplied by our model's predicted asymptotic skill level, $\hat{x}_{\mathrm{k}}=s_{\mathrm{k}} B_{\mathrm{b}} x_{\mathrm{b}}^{*}\left(t_{\mathrm{a}}\right) / B_{\mathrm{k}}$. 